\title{
A Symmetric Family of Yang-Mills Fields
}

\section{Lorenzo Sadun}

Department of Mathematics, University of Texas, Austin, Texas 78712, USA

E-mail address: sadun@math.utexas.edu

Received: 5 February 1993/in revised form: 29 October 1993

\begin{abstract}
We examine a family of finite energy $S O(3)$ Yang-Mills connections over $S^{4}$, indexed by two real parameters. This family includes both smooth connections (when both parameters are odd integers), and connections with a holonomy singularity around 1 or 2 copies of $R P^{2}$. These singular YM connections interpolate between the smooth solutions. Depending on the parameters, the curvature may be self-dual, anti-self-dual, or neither. For the (anti)self-dual connections, we compute the formal dimension of the moduli space. For the non-self-dual connections we examine the second variation of the Yang-Mills functional, and count the negative and zero eigenvalues. Each component of the non-self-dual moduli space appears to consist only of conformal copies of a single solution.
\end{abstract}

\section{Introduction and Statement of Results}

1.1 Main Results. Until recently, the phrase "Yang-Mills theory in four dimensions" essentially meant the study of smooth solutions to the (anti) self-duality equations

$$
* F= \pm F,
$$

where $F$ is the curvature of a connection $A$, usually with gauge group $S U(2)$ or $S O(3)$, on a bundle over a Riemannian 4-manifold $M$, which may or may not have a boundary. The moduli space of such solutions, up to gauge invariance, gives topological information about $M$, a fact which was exploited by Donaldson and others to make tremendous progress in the topology of 4-manifolds (see [DK] for an overview).

In recent years the field has expanded in two directions. First, there is the study of nonself-dual Yang-Mills connections. These are solutions to the full Yang-Mills equations,

$$
d_{A}^{*} F=0,
$$

This work was partially supported by an NSF Mathematical Sciences Postdoctoral Fellowship 
whose curvature is neither self-dual nor anti-self-dual. It was generally assumed that, for gauge group $S U(2)$ or $S O(3)$, such solutions did not exist on bundles over the 4-sphere, until Sibner, Sibner, and Uhlenbeck [SSU] constructed such solutions on the trivial $S U(2)$ bundle over $S^{4}$ in 1988.

The other, and more important, extension has been to consider finite-energy (anti)self-dual connections with a holonomy singularity. The first example was found by Forgacs, Horvath, and Palla [FHP1, FHP2]. The first general results were the regularity theorems of Sibner and Sibner [SiSi1, SiSi2]. The subject gained attention when Kronheimer and Mrowka [K, KM] used instantons with holonomy to study embedding of 2-manifolds in 4-manifolds. Since then others have tried to advance the general theory of such instantons [R1, R2], but there are many tricky questions that are still not well understood.

In this paper we consider a family of solutions to the full $S O$ (3) Yang-Mills equations (1.2) on $X=S^{4} \backslash\left\{S_{+} \cup S_{-}\right\}$, where $S_{+}$and $S_{-}$are linked embedded copies of $R P^{2}$. This family contains solutions both with and without holonomy, and whose curvature is self-dual, anti-self-dual, and non-self-dual. Although only a few of these solutions have been written in closed form [BoSe], these solutions can all be well-approximated numerically, and their asymptotic behavior as the indexing parameters get large is well-understood [SS3]. It is hoped that these examples will help researchers build an intuition for how singular and nonself-dual YM connections behave.

The solutions are indexed by two positive real numbers $(r, t)$, which reflect the holonomy of the connection around $S_{+}$and $S_{-}$, respectively. When $r$ and $t$ are odd integers, the holonomy about $S_{ \pm}$is trivial, and the solution can be smoothly extended to all of $S^{4}$. These smooth solutions were previously discussed, for gauge group $S U(2)$, in [SS1, SS2, SS3, BoMo, Bor].

The solutions we consider are all symmetric with respect to an $S O(3)$ action on $S^{4}$. By considering only symmetric connections, we reduce the Yang-Mills equations and the self-duality equations to a system of ODEs, which we call the reduced YM (or self-duality) equations. Using ODE methods, we then prove:

Theorem 1.1. For each pair of non-negative real numbers $(r, t)$ there exists a YangMills connection on the trivial bundle over $X$ with the following properties:

i. The holonomy around $S_{+}$is (conjugate to) $\exp (i \pi(r+1))$. The holonomy around $S_{-}$is (conjugate to) $\exp (i \pi(t+1))$.

ii. The integral over $X$ of the Chern-Weil form (a.k.a. the fractional Chern number) is $\left(r^{2}-t^{2}\right) / 8$.

iii. If $r$ and $t$ are both greater than 1 , or both strictly between 0 and 1 , then the solution has non-self-dual curvature.

iv. If $r \geqq 1 \geqq t$, or if $t=0$, then the solution has anti-self-dual curvature. If $t \geqq 1 \geqq r$,or if $r=0$, then the solution has self-dual curvature.

Some of these results are not new. The dimensional reduction from 4 to 1 dimensions was developed by Urakawa [U], and was applied by Bor and Montgomery [BoMo, Bor] to this particular symmetry. In [SS1, SS2] this method was used to prove the existence of non-self-dual YM connections with $r>1, t>1$. The case $r=1, t=$ odd has been studied by Bor and Segert [BoSe] using an equivariant ADHM construction [ADHM]. 
By the Peter-Weyl theorem, deformations of the solutions can be decomposed into irreducible representations of the symmetry group $S O(3)$. The linearized Yang-Mills equations for these deformations reduce to a countable collection of ODE systems, one for each representation. By counting the solutions to these ODEs, with appropriate boundary conditions, we deduce

Theorem 1.2. If $r \geqq 3$ and $t=1$, then the number of linearly independent regular solutions to the linearized anti-self-duality equations (i.e. the formal dimension of the moduli space) generically equals

$$
\{r\}^{2}-4
$$

where $\{x\}$ denotes the greatest odd integer less than or equal to $x$. If $r \geqq 1 \geqq t$ and either $r<3$ or $t<1$, then the dimension generically equals zero.

The mechanism by which the dimension of the moduli space jumps is extremely simple. Let $d$ be the distance from a point to the singular set $S_{+}$. The natural boundary conditions at $S_{+}$are that certain components of the connection remain bounded as $d \rightarrow 0$ if the holonomy around $S_{+}$is trivial, and go to zero as $d \rightarrow 0$ if the holonomy is non-trivial. Solving the linearized anti-self-duality equations in a particular representation of $S O(3)$ gives solutions that behave like $d^{(r-M) / 2}$, where $M$ is an odd integer that depends on the representation. If $r>M$ the solution goes to zero as $d \rightarrow 0$, and so satisfies the boundary conditions. If $r=M$ then the solution approaches a finite limit at $d=0$, and so is still admissible. However, if $r<M$ the solution blows up at $S_{+}$and is disallowed. Counting the contributions of the representations that have $M \leqq r$, we get formula (1.3).

The boundary conditions that lead to formula (1.3) are natural but not unique. When $r$ and $t$ are not both odd integers, one has a choice as to how big a space of connections to consider, and how big the corresponding gauge group should be. By making these choices in a reasonable but non-standard way, one can get boundary conditions weaker than those that lead to formula (1.3). These alternate boundary conditions, which we call weak regularity, give a moduli space with a slightly different dimension.

Theorem 1.3. If $r \geqq 3$ and $t=1$, then the number of linearly independent weakly regular solutions to the linearized anti-self-duality equations generically equals

$$
(\{r\}+1)^{2}-4
$$

when $r$ is not an odd integer, and $r^{2}-4$ when $r$ is an odd integer. If $t<1$, then the dimension generically equals

$$
(\{r\}+1)^{2}-1
$$

when $r$ is not an odd integer, and $r^{2}-1$ when $r$ is an odd integer.

For $t=1$, these results concur with the general results of Kronheimer and Mrowka [KM], who studied anti-self-dual connections with orientable singular sets. As in [KM], the discontinuities in the dimension of the moduli space all occur when the $(S O(3))$ holonomy is trivial. Notice also that the dimension (1.4) is always even. This suggests the possibility of computing a $Z_{2}$-valued Donaldson polynomial on the fundamental class of $S_{-}\left(S_{-}\right.$is a deformation retract of $\left.S^{4}-S_{+}\right)$.

Direct comparison with [KM] is complicated by the fact that our singular set $S_{+}$is non-orientable. The $C^{2}$ bundle associated to our principal bundle does not 
split into a sum of line bundles near $S_{+}$. It splits locally, but parallel transport along a generator of $\pi_{1}\left(S_{+}\right)$interchanges the two factors. To get a reasonable "monopole number" $l$, we must lift to the double cover of $S_{+}$, compute the first Chern number there, and then divide by two. If we take the holonomy parameter $\alpha$ to be half the fractional part of $(r+1) / 2$, then $l=\{r\} / 2$ and the "instanton number" $k$ equals $\left(\{r\}^{2}-1\right) / 8$. If we take $\alpha$ to be $1 / 2$ minus the fractional part of $(r+1) / 2$, then $l=-\{r+2\} / 2$ and $k=\left(\{r+2\}^{2}-1\right) / 8$. In either case, $l$ is not an integer.

With these identifications, the formula (1.4) for weakly regular solutions gives the same dimension as [KM]'s formula (1.6), where $S_{+}$is understood to have genus $1 / 2$ and self-intersection number -2 . Similarly, our formula for the energy agrees with [KM]'s formula (1.7).

The second variation of the Yang-Mills functional (the YM Hessian) also decomposes as a direct sum of operators, one for each irreducible representation of $S O(3)$. This is important for the non-self-dual connections, as it allows us to count the negative and zero eigenvalues of the Hessian, one representation at a time. When $r$ and $t$ are small odd integers, numerical diagonalization of the Hessian indicates that

$$
\text { Index of Hessian of }(r, t) \text { connection }=\frac{(r-1)(t-1)(r+t-2)}{2} .
$$

In the (anti)self-dual cases this index is of course zero. In the non-self-dual cases, this index greatly exceeds the lower bound found by Taubes [T1], and is always a multiple of 8 .

For the smooth non-self-dual cases we also find that, after gauge fixing,

$$
\text { Nullity of Hessian of }(r, t) \text { connection }=12 \text {, }
$$

regardless of the values of $r$ and $t$. These zero modes all come from conformal symmetry, so there appears to be no interesting structure to each component of the non-self-dual moduli space.

1.2 Outline of Paper. In Sects. 2.1-2.3 we quickly review the dimensional reduction and the derivation of the reduced Yang-Mills and reduced self-duality equations. This is largely taken from [SS2], with appropriate changes for having symmetry group (and gauge group) $S O(3)$ rather than $S U(2)$. These sections are terse and the proofs have largely been omitted. For a more detailed discussion of this construction the reader is referred to [SS2].

In the remainder of Sect. 2 we discuss the decomposition of a deformation of an equivariant connection into representations of $S O(3)$. We also consider the difference between $S O(3)$ connections and $S U(2)$ connections. Although we consistently work with $S O(3)$ in this paper, almost all the results apply equally well to $S U(2)$.

In Sect. 3 we study the reduced (anti)self-duality equations and prove Theorem 1.1

In Sect. 4 we study the linearized anti-self-duality equations and prove Theorems 1.2 and 1.3 .

In Sect. 5 we study non-self-dual solutions to the Yang-Mills equations, and numerically investigate the index and nullity of the Yang-Mills Hessian, arriving at formulas (1.6) and (1.7). 


\section{Symmetric Gauge Fields}

2.1 Symmetry on $S^{4}$. We consider the symmetry group $G=S O(3)$. Let $K_{1}, K_{2}$, $K_{3}$ be the matrices

$$
K_{1}=\left(\begin{array}{ccc}
0 & 0 & 0 \\
0 & 0 & -1 \\
0 & 1 & 0
\end{array}\right), \quad K_{2}=\left(\begin{array}{ccc}
0 & 0 & 1 \\
0 & 0 & 0 \\
-1 & 0 & 0
\end{array}\right), \quad K_{3}=\left(\begin{array}{ccc}
0 & -1 & 0 \\
1 & 0 & 0 \\
0 & 0 & 0
\end{array}\right)
$$

and let $l_{i}$, (or $r_{i}$ ) be the generator of right (or left)-translations by $\exp \left(t K_{i}\right)$. The vector fields $l_{i}$ are left-invariant (since right-translations commute with left-translations) and form a basis for the Lie algebra of $G$. It is easy to check that $\left[l_{1}, l_{2}\right]=l_{3}$, etc. If we let $\beta^{i}$ denote the 1 -form dual to $l_{i}$, then the Maurer-Cartan equations are $d \beta^{1}=-\beta^{2} \wedge \beta^{3}$, etc. The Laplacian on $G, \Delta=-\sum_{i} r_{i}^{2}=-\sum_{i} l_{i}^{2}$, is both left- and right-invariant.

Let $I$ denote the open interval $(0, \pi / 3)$, and let $\bar{I}$ denote the closed interval $[0, \pi / 3]$. Let $k_{i}=\exp \left(\pi K_{i}\right)$. We also define some subgroups of $G$, letting $L_{i}=$ $\left\{\exp \left(t K_{i}\right) ; t \in[0,2 \pi)\right\}$, and letting $\Gamma=\left\{1, k_{1}, k_{2}, k_{3}\right\}$.

Let $V \simeq \mathbb{R}^{5}$ be the space of symmetric, traceless, real $3 \times 3$ matrices $Q$, with inner product $\left\langle Q, Q^{\prime}\right\rangle=\frac{1}{2} \operatorname{Tr}\left(Q Q^{\prime}\right)$. It is useful to work with an explicit orthonormal basis. Let

$$
\begin{gathered}
Q_{0}=\frac{1}{\sqrt{3}}\left(\begin{array}{ccc}
-1 & 0 & 0 \\
0 & -1 & 0 \\
0 & 0 & 2
\end{array}\right), Q_{1}=\left(\begin{array}{lll}
0 & 0 & 1 \\
0 & 0 & 0 \\
1 & 0 & 0
\end{array}\right) \\
Q_{2}=\left(\begin{array}{lll}
0 & 0 & 0 \\
0 & 0 & 1 \\
0 & 1 & 0
\end{array}\right), Q_{3}=\left(\begin{array}{ccc}
1 & 0 & 0 \\
0 & -1 & 0 \\
0 & 0 & 0
\end{array}\right), Q_{4}=\left(\begin{array}{lll}
0 & 1 & 0 \\
1 & 0 & 0 \\
0 & 0 & 0
\end{array}\right) .
\end{gathered}
$$

A matrix $g$ in $G=S O(3)$ acts on $V$ isometrically by conjugation, $g(Q) \rightarrow g Q g^{-1}$. The unit sphere $S^{4} \subset V$ inherits this $S O(3)$ action.

Since all matrices in $V$ are diagonalizable, it is not hard to check that every $Q \in S^{4}$ is related by the group action to a unique $Q_{\theta}=\cos (\theta) Q_{0}+\operatorname{Sin}(\theta) Q_{3}$ with $\theta \in \bar{I}$. For $\theta \in I$, the isotropy group of $Q_{\theta}$ is $\Gamma$, so the orbit of $Q_{\theta}$ is three dimensional, and in particular is isomorphic to $G / \Gamma$. The isotropy group of $Q_{0}$, which we denote $J_{0}$, is generated by $\Gamma$ and $L_{3}$, while $J_{\pi / 3}$, the isotropy group of $Q_{\pi / 3}$, is generated by $\Gamma$ and $L_{2}$. The orbits of $Q_{0}$ and $Q_{\pi / 3}$ are isomorphic to $R P^{2}$.

Let $X \subset S^{4}$ be the union of the three-dimensional orbits, and let $Y=I \times G$. Since each orbit is isomorphic to $G / \Gamma$, we have $Y / \Gamma \sim X$, with the projection map

$$
\begin{aligned}
& s: Y \rightarrow X, \\
& (\theta, g) \rightarrow g Q_{\theta} g^{-1} .
\end{aligned}
$$

It is useful to think of functions on $X$ as $\Gamma$-invariant functions on $Y$. 
The standard metric on $X$ as a subset of $S^{4}$ pulls back under $s$ to give a metric on $Y$. The vectors fields $\left\{\partial / \partial \theta, l_{1}, l_{2}, l_{3}\right\}$ form a basis for $T Y$, and are orthogonal but not orthonormal. $\partial / \partial \theta$ is a unit vector, but $l_{i}$ has norm $f_{i}(\theta)$, where

$$
f_{1}(\theta)=2 \sin (\pi / 3+\theta) ; \quad f_{2}(\theta)=2 \sin (\pi / 3-\theta) ; \quad f_{3}(\theta)=2 \sin (\theta) .
$$

Since $d \theta$ and $f_{i} \beta^{i}$ form an orthonormal basis of 1 -forms, it is easy to write down the volume form on $Y$,

$$
\eta=f_{1} f_{2} f_{3} \beta^{1} \wedge \beta^{2} \wedge \beta^{3} \wedge d \theta
$$

and the action of the Hodge dual on 2-forms ${ }^{1}$ :

$$
*\left(d \theta \wedge \beta^{1}\right)=-G_{1} \beta^{2} \wedge \beta^{3} ; \quad *\left(d \theta \wedge \beta^{2}\right)=-G_{2} \beta^{3} \wedge \beta^{1} ; \quad *\left(d \theta \wedge \beta^{3}\right)=-G_{3} \beta^{1} \wedge \beta^{2},
$$

where

$$
G_{1} \equiv \frac{f_{2} f_{3}}{f_{1}} ; \quad G_{2} \equiv \frac{f_{3} f_{1}}{f_{2}} ; \quad G_{3} \equiv \frac{f_{1} f_{2}}{f_{3}}
$$

2.2 Bundle Structures. We now construct $S O(3)$ principal bundles over $Y$, over $X$, and over $S^{4}$. SO(3) is both the symmetry group of the base and of the fiber. We denote the symmetry group of the base by $G=S O(3)$, and the gauge group by $H=S O(3)$. Let $P_{Y}=Y \times H$ be the trivial bundle. $H$ acts on the right,

$$
(\theta, g, h) \mapsto\left(\theta, g, h h^{\prime}\right), \quad h^{\prime} \in H,
$$

while $G$ acts on the left,

$$
(\theta, g, h) \mapsto\left(\theta, g^{\prime} g, h\right), \quad g^{\prime} \in G .
$$

These two actions obviously commute. We next define an equivalence relation

$$
(\theta, g, \gamma h) \sim(\theta, g \gamma, h), \quad \gamma \in \Gamma,
$$

and define $P_{X}=P_{Y} / \sim P_{X}$ is a principal $S O(3)$ bundle with base space $Y / \Gamma=X$.

For considering connections with holonomy, $P_{X}$ is all we need. However, we also wish to consider the case where the holonomy is trivial and the bundle and connection can be extended to all of $S^{4}$. We start with the trivial bundle $P_{\bar{Y}}=\bar{I} \times G \times H$, and mod out by an extension of the equivalence relation $\sim$. The equivalence relations at $\theta=0, \pi / 3$ involve the isotropy subgroups $J_{0}$ and $J_{\pi / 3}$, so the base space for our bundle will be $X \cup\left\{G / J_{0}\right\} \cup\left\{G / J_{\pi / 3}\right\}=X \cup S_{+} \cup S_{-}=S^{4}$.

Let $r$ and $t$ be odd integers. We define the equivalence generated by

$$
\begin{aligned}
(\theta, g \gamma, h) & \sim(\theta, g, \gamma h), \quad \gamma \in \Gamma, \theta \in \bar{I}, \\
(0, g \gamma, h) & \sim\left(0, g, \gamma^{-r} h\right), \quad \gamma \in L_{3}, \\
(\pi / 3, g \gamma, h) & \sim\left(\pi / 3, g, \gamma^{-t} h\right), \quad \gamma \in L_{2} .
\end{aligned}
$$

Since $k_{3}$ is in both $\Gamma$ and $L_{3}$, we need $r$ to be odd for this definition of $\sim$ to be consistent. Similarly, $t$ must also be odd, as $k_{2}$ is in both $\Gamma$ and $L_{2}$.

${ }^{1}$ In [SS2] the orientation on $S^{4}$ and the sign of the Chern number were chosen opposite to standard conventions. As a result, some of the formulas in this paper differ in sign from those of [SS2]. 
We let $P_{(r, t)}=P_{\bar{Y}} / \sim$. To see that this is in fact a bundle over $S^{4}$, we construct local sections. Away from the orbits of $Q_{0}$ and $Q_{\pi / 3}$ we have the canonical section

$$
\kappa:(\theta, g) \mapsto(\theta, g, 1) .
$$

Next we construct a local section over a neighborhood $U$ of $Q_{0}$. The local product structure near $Q_{\pi / 3}$ is entirely analogous.

We first specify the neighborhood $U$. Let $D$ be the open disk

$$
D=\left\{\left(y_{1}, y_{2}\right) \in \mathbb{R}^{2} \mid y_{1}^{2}+y_{2}^{2}<(\pi / 4)^{2}\right\},
$$

and let $S=\mathbb{R}(\bmod 2 \pi)$ be a circle. The map

$$
\begin{aligned}
\psi: D \times S & \rightarrow G \\
\left(y_{1}, y_{2}, y_{3}\right) & \mapsto \exp \left(y_{1} K_{1}+y_{2} K_{2}\right) \exp \left(y_{3} K_{3}\right)
\end{aligned}
$$

is a diffeomorphism of $D \times S$ onto its image, which we call $N$. $N$ is clearly invariant under right translations by $L_{3}$. Letting $M=[0, \pi / 6) \times N \subset Y$, we take $U$ to be the image of $M \subset \bar{Y}$ under the map $s$ (Eq. 2.3).

To construct a section, we first define a map $\varphi: N \rightarrow H$,

$$
\varphi(g) \equiv \exp \left(r y_{3} K_{3}\right) \in H,
$$

The section

$$
\begin{aligned}
& \delta: \bar{M} \rightarrow P_{r, t} \\
& (\theta, g) \mapsto(\theta, g, \varphi(g))
\end{aligned}
$$

passes to the quotient, giving a local section of $P_{r, t}$ over the neighborhood $U$ of $Q_{0}$ in $S^{4}$.

2.3 Dimensional Reduction. A connection on $P_{X}$ is equivalent to a $\Gamma$-invariant connection on $P_{Y}$. It must therefore take the form

$$
A=\sum_{i, j} \alpha_{i j}(\theta, g) \beta^{i} \otimes l_{j}+\sum_{i} \gamma_{i}(\theta, g) d \theta \otimes l_{i},
$$

where $\alpha_{i j}$ transforms under $\Gamma$ in the same way as $\beta^{i} \otimes l_{j}$, and $\gamma_{i}$ transforms like $l_{i}$. We call functions that transform like $l_{1}$, e.g. $\alpha_{32}, \alpha_{23}$, and $\gamma_{1}$, "x-type." Functions like $\alpha_{31}, \alpha_{13}$, and $\gamma_{2}$, which transform like $l_{2}$, are called "y-type.,' and functions like $\alpha_{21}, \alpha_{12}$, and $\gamma_{3}$, which transform like $l_{3}$, are called "z-type." Replacing $g$ with $g k_{1}$ flips the sign of the y-type and z-type functions, replacing $g$ with $g k_{2}$ flips the sign of the x-type and z-type functions, and replacing $g$ with $g k_{3}$ flips the sign of the $\mathrm{x}$-type and y-type functions. The functions $\alpha_{11}, \alpha_{22}$, and $\alpha_{33}$ are invariant under $\Gamma$.

We next restrict our attention to $G$-equivariant connections on $P_{X}$. This means that the functions $\alpha_{i j}$ and $\gamma_{i}$ depend only on $\theta$, not on $g$, and so are necessarily $\Gamma$-invariant. As a result, all the x-type, y-type, and z-type functions must be identically zero. A $G$-equivariant connection on $P_{X}$ therefore takes the form

$$
A=-a_{1}(\theta) \beta^{1} \otimes l_{1}-a_{2}(\theta) \beta^{2} \otimes l_{2}-a_{3}(\theta) \beta^{3} \otimes l_{3} .
$$

We refer to the triplet of functions $a=\left(a_{1}, a_{2}, a_{3}\right)$ as a reduced connection. 
Given an equivariant connection $A$, the curvature $F$ is easily computed:

$$
F=\left(\left(a_{1}+a_{2} a_{3}\right) \beta^{2} \wedge \beta^{3}-a_{1}^{\prime} d \theta \wedge \beta^{1}\right) \otimes l_{1}+(\text { cyclic }),
$$

where' denotes $d / d \theta$, and (cyclic) denotes the other cyclic permutations of the indices $(1,2,3)$. By Eq. (2.6) the (anti)self-duality equations are then

$$
a_{1}^{\prime}= \pm \frac{\left(a_{1}+a_{2} a_{3}\right)}{G_{1}}, \quad a_{2}^{\prime}= \pm \frac{\left(a_{2}+a_{1} a_{3}\right)}{G_{2}}, \quad a_{3}^{\prime}= \pm \frac{\left(a_{3}+a_{1} a_{2}\right)}{G_{3}},
$$

where + denotes self-duality and - denotes anti-self-duality.

From $F$ we compute the Yang-Mills functional, with the result

$$
\begin{aligned}
\mathscr{Y} \mathscr{M}(A)=S(a)= & \pi^{2} \int_{0}^{\pi / 3} d \theta\left[\left(a_{1}^{\prime}\right)^{2} G_{1}+\left(a_{2}^{\prime}\right)^{2} G_{2}+\left(a_{3}^{\prime}\right)^{2} G_{3}\right. \\
& \left.+\frac{\left(a_{1}+a_{2} a_{3}\right)^{2}}{G_{1}}+\frac{\left(a_{2}+a_{1} a_{3}\right)^{2}}{G_{2}}+\frac{\left(a_{3}+a_{1} a_{2}\right)^{2}}{G_{3}}\right] .
\end{aligned}
$$

For equivariant connections, the Yang-Mills equations $d_{A}^{*} F=0$ are equivalent to the Euler-Lagrange equations for the one-dimensional functional $S(a)$ [SS2].

Since $G_{3}(\theta)$ is bounded away from zero near $\theta=0$, a finite-action reduced connection must have $a_{3}^{\prime}$ integrable near $\theta=0$, so the boundary value $r \equiv a_{3}(0)$ is well-defined. Similarly, $t=a_{2}(\pi / 3)$ is well-defined. Also, since $G_{1}$ and $G_{2}$ have zeroes at $\theta=0$, finite-action reduced connections must have

$$
\lim _{\theta \rightarrow 0}\left[a_{1}(\theta)+a_{2}(\theta) a_{3}(\theta)\right]=\lim _{\theta \rightarrow 0}\left[a_{2}(\theta)+a_{1}(\theta) a_{3}(\theta)\right]=0 .
$$

If $r \neq \pm 1$, these conditions imply that both $a_{1}(0)$ and $a_{2}(0)$ exist and equal zero. Similarly, if $t \neq \pm 1$ then $a_{1}(\pi / 3)=a_{3}(\pi / 3)=0$. We call a finite-action reduced connection with $a_{3}(0)=r$ and $a_{2}(\pi / 3)=t$ a reduced $(r, t)$ connection.

The boundary values $r$ and $t$ are related to the holonomy of the connection $A$ around $S_{+}$and $S_{-}$, respectively. For fixed $\theta \in I$, we consider the path $\left(\theta, \exp \left(\tau K_{3}\right)\right)$ on $Y$, where $\tau$ runs from 0 to $\pi$. This path projects to a closed loop on $X$, from $Q_{\theta}$ to itself. The tangent vector $d / d \tau$ along the path is $l_{3}$, so parallel transport is given by intergrating $a_{3}$ along the path. The point $(\theta, 1,1)$ in $P_{Y}$ is transported to $\left(\theta, \exp \left(\pi K_{3}\right), \exp \left(\pi a_{3}(\theta) K_{3}\right)\right)$. Under the identification (2.10), this is equivalent to $\left(\theta, 1, \exp \left(\left(1+a_{3}(\theta)\right) \pi K_{3}\right)\right)$. Taking the limit as $\theta \rightarrow 0$, we see that the holonomy around $S_{+}$is $\exp \left((r+1) \pi K_{3}\right)$, which is trivial if and only if $r$ is an odd integer. Similarly, the holonomy around $S_{-}$is $\exp \left((t+1) \pi K_{2}\right)$, which is trivial if and only if $t$ is an odd integer.

Finally, we compute $C_{2}$, the integral of the second Chern-Weil form:

$$
C_{2} \equiv \int_{X} \frac{\operatorname{Tr}(F \wedge F)}{8 \pi^{2}}
$$

Since $X$ is an open manifold, $C_{2}$ need not be an integer, depending on the holonomy around the two-dimensional singular sets [FHP1, FHP2]. From Eq. (2.19) we immediately get

$$
\begin{aligned}
\operatorname{Tr}(F \wedge F) & =\left(a_{1}^{\prime}\left(a_{1}+a_{2} a_{3}\right)+\text { cyclic }\right) d \theta \wedge \beta^{1} \wedge \beta^{2} \wedge \beta^{3} \\
& =\frac{1}{2} d\left(a_{1}^{2}+a_{2}^{2}+a_{3}^{2}+2 a_{1} a_{2} a_{3}\right) \wedge \beta^{1} \wedge \beta^{2} \wedge \beta^{3} .
\end{aligned}
$$


Integrating first over the symmetry group and then over $I$, we get

$$
C_{2}=\int_{I} \frac{-1}{8} d\left(a_{1}^{2}+a_{2}^{2}+a_{3}^{2}+2 a_{1} a_{2} a_{3}\right)=\operatorname{CS}(0)-\operatorname{CS}(\pi / 3),
$$

where

$$
\operatorname{CS}(\theta)=\left(a_{1}(\theta)^{2}+a_{2}(\theta)^{2}+a_{3}(\theta)^{2}+2 a_{1}(\theta) a_{2}(\theta) a_{3}(\theta)\right) / 8
$$

is the reduced Chern-Simons function.

If $r \neq \pm 1$, then $a_{1}(0)=a_{2}(0)=0$, and $\operatorname{CS}(0)=r^{2} / 8$. If $r= \pm 1$, then $\operatorname{CS}(0)=$ $r^{2} / 8+\lim _{\theta \rightarrow 0}\left(a_{1}(\theta)+r a_{2}(\theta)\right)^{2} / 8$. This second term is zero (Eq. 2.22), so we still have $\mathrm{CS}(0)=r^{2} / 8$. Similarly, $\mathrm{CS}(\pi / 3)=t^{2} / 8$, and so $C_{2}=\left(r^{2}-t^{2}\right) / 8$.

The reduced self-duality equations (2.20), the Yang-Mills functional (2.21), and the reduced Chern-Simons functional (2.26) are left unchanged if we flip the signs of two of the three functions $\left(a_{1}, a_{2}, a_{3}\right)$. This is a consequence of gauge invariance, since global gauge transformations by $k_{1}, k_{2}$, or $k_{3}$ flip the signs of $a_{2}$ and $a_{3}$, $a_{1}$ and $a_{3}$, or $a_{1}$ and $a_{2}$, respectively. Without loss of generality, we can therefore restrict our attention to non-negative $r$ and $t$.

2.4 Classifying deformations. Once we have established the existence of equivariant Yang-Mills connections, we will wish to consider infinitesimal deformations of these solutions. These deformations need not be equivariant, and can take the general form

$$
\delta A=\sum_{i, j} \alpha_{i j}(\theta, g) \beta^{i} \otimes l_{j}+\sum_{i} \gamma_{i}(\theta, g) d \theta \otimes l_{i}
$$

Since the metric on $X$ and original solution $A$ are invariant under the action of $G$, the solutions of the linearized self-duality equations

$$
* \delta F= \pm \delta F
$$

and the eigenspaces of the Yang-Mills Hessian can be decomposed into irreducible representations of $S O(3)$, and in particular can be chosen to be eigenfunctions of the Laplacian.

An orthonormal basis for $L^{2}(S O(3))$ is given by the functions

$$
\Psi_{l, m_{r}, m_{l}}(g), \quad l=0,1,2, \ldots, \quad m_{r}=-l, 1-l, \ldots, l, \quad m_{l}=-l, 1-l, \ldots, l,
$$

where $\Psi_{l, m_{r}, m_{l}}$ is an eigenfunction of the Laplacian with eigenvalue $l(l+1)$, of $-i r_{3}$ with eigenvalue $m_{r}$, and of $-i l_{3}$ with eigenvalue $m_{l}$. Since right-translations and left-translations commute, $l$ and $m_{l}$ are preserved by left-translations, while $l$ and $m_{r}$ are preserved by right-translations.

As a result, we may fix $l$ and $m_{l}$ while looking for eigenvalues of the Hessian and solutions of the linearized self-duality equations. Moreover, for fixed $l$ either a solution exists for all $m_{l}$ or for none. We can therefore do our calculations for a single value of $m_{l}$, and then multiply the multiplicity by $2 l+1$ to account for the other values. We choose a useful basis as follows:

Proposition 2.1. There exist real functions $\Psi_{l, m}^{ \pm}, m=1,2, \ldots, l$ and $\Psi_{l, 0}$, which, together with their left translates, span the $l^{\text {th }}$ eigenspace of the Laplacian on 
SO(3), and have the following properties:

1. If $m$ is odd, then $\Psi_{l, m}^{+}$is an x-type function and $\Psi_{l, m}^{-}$is $y$-type.

2. If $m$ is even, then $\Psi_{l, m}^{+}$is a $z$-type function and $\Psi_{l, m}^{-}$is invariant under $\Gamma$.

3. $\Psi_{l, 0}$ is z-type if $l$ is odd and invariant if $l$ is even.

4.

$$
l_{3} \Psi_{l, m}^{ \pm}= \pm m \Psi_{l, m}^{\mp}
$$

Proof. We start with the functions $\Psi_{l, 0, m}$. Their span, which we call $W$, is a $2 l+1$ dimensional representation of the right-action so $S O(3)$. Together with their lefttranslates, the $\Psi_{l, 0, m}$ 's span the $l^{\text {th }}$ eigenspace of the Laplacian. Since $\Psi_{l, 0, m}$ is an eigenfunction of $l_{3}$ with eigenvalue $i m$, we have $\Psi_{l, 0, m}\left(g \exp \left(\pi K_{3}\right)\right)=$ $(-1)^{m} \Psi_{l, 0, m}(g)$. Thus for $m$ odd $\Psi_{l, 0, m}$ must be a linear combination of x-type and y-type functions, and for $m$ even $\Psi_{l, 0, m}$ must be a linear combination of z-type and invariant functions.

Because of its own transformation properties under $\Gamma, l_{3}$ maps x-type and $\mathrm{y}$-type functions to each other (e.g. if $\psi$ is $\mathrm{x}$-type then $l_{3} \psi$ is y-type) and maps $\mathrm{z}$-type and invariant functions to each other. Since $\Psi_{l, 0, m}$ is an eigenfunction of $l_{3}, l_{3}$ must map the x-type (z-type) and y-type (invariant) parts of $\Psi_{l, 0, m}$ to multiples of each other. This also shows that none of these parts are zero. The projection $P_{+}$onto the $\mathrm{x}$-type and z-type parts of a function can be written in terms of right-translations,

$$
\left(P_{+} \psi\right)(g)=\left(\psi(g)-\psi\left(g k_{2}\right)\right) / 2,
$$

so for any $\psi \in W, P_{+} \psi \in W$.

For $m>0$, let $\Psi_{l, m}^{+}=P_{+} \Psi_{l, 0, m}$, rescaled to have unit norm, and let $\Psi_{l, m}^{-}=l_{3} \Psi_{l, m}^{+} / m$. Since $l_{3}^{2}$ commutes with $P_{+}, l_{3}^{2} \Psi_{l, m}^{+}=-m^{2} \Psi_{l, m}^{+}$, and Eq. (2.30) follows. It also follows that $\Psi_{l, m}^{-}$has unit norm. To complete our basis we take $\Psi_{l, 0}=\Psi_{l, 0,0}$.

Any two of these functions either correspond to different eigenvalues of $l_{3}^{2}$ or to different transformation properties under $\Gamma$, and so must be orthogonal. Our collection, which contains $2 l+1$ elements, is therefore an orthonormal basis for $W$.

$\Psi_{l, 0}$ must be either z-type or invariant. It cannot be a combination of both, or else we could decompose it into $P_{l, 0}^{ \pm}$and end up with $2 l+2$ linearly independent functions in $W$. To see which type $\Psi_{l, 0}$ is, we use a simple counting argument.

The dimension of the x-type subspace of $W$ equals the dimension of the y-type subspace, since $l_{3}$ maps x-type and z-type functions to each other. This is also the dimension of the z-type subspace, as $l_{2}$ maps $x$-type and z-type functions to each other. Of the basis elements $\Psi_{l, m}^{ \pm}$, there are [l/2] z-type functions ( $m$ even) and $[(l+1) / 2] x$-type functions $(m$ odd), where $[x]$ denotes the integer part of $x$. To keep the total number of $\mathrm{x}$-type and z-type basis elements equal, $\Psi_{l, 0}$ must be $\mathrm{z}$-type if $l$ is odd, and invariant if $l$ is even.

$2.5 S O(3)$ vs. $S U(2)$. We have done our construction for symmetry group $G=S O(3)$ and gauge group $H=S O(3)$. However, the construction was first carried out for $S U(2)$, and is nearly identical.

In the $S U(2)$ case, we take symmetry and gauge groups $\tilde{G}=\tilde{H}=S U(2)$, and think of $S U(2)$ as the set of unit quaternions. Since $\widetilde{G}$ is the double cover of $G$, the action of $G$ on $S^{4}$ lifts to an action of $\tilde{G}$. The isotropy group of $Q_{\theta}$ is now the 8 element group $\widetilde{\Gamma}=\{ \pm 1, \pm i, \pm j, \pm k\}$. To get a principal bundle over $X$ we define 
the bundle $P_{\tilde{Y}}=I \times \tilde{G} \times \tilde{H}$ and $\bmod$ out by the equivalence relation (2.10), where now we think of $g, h, \gamma$ as arbitrary elements of $\tilde{G}, \tilde{H}$, and $\tilde{\Gamma}$ respectively.

The dimensional reduction procedure is identical to that of Sect. 2.3, with Eqs. (2.17) through (2.25) remaining true. The only difference is in the interpretations of the two numbers $(r, t)$. For $S U(2)$, the holonomy around $S_{+}$is $\exp (\pi(r+1) k / 2)$, rather than $\exp \left(\pi(r+1) K_{3}\right)$, and the holonomy around $S_{-}$is $\exp (\pi(t+1) j / 2)$. Note that the holonomy for $r$ is not conjugate to that for $-r$, so an $(r, t) S U(2)$ connection cannot be gauge-equivalent to a $(-r, t)$ connection, an $(r,-t)$ connection, or a $(-r,-t)$ connection.

Although the (4-dimensional) connections are not gauge-equivalent, the onedimensional equations are still invariant under a pair of sign flips. If $a=\left(a_{1}, a_{2}, a_{3}\right)$ is a reduced connection, then the four reduced connections $\left(a_{1}, a_{2}, a_{3}\right)$, $\left(a_{1},-a_{2},-a_{3}\right),\left(-a_{1}, a_{2},-a_{3}\right)$ and $\left(-a_{1},-a_{2}, a_{3}\right)$ all have the same curvature (up to sign), have the same Chern number, are all self-dual if any one is, and are all Yang-Mills if any one is. As $S O(3)$ connections they are all gauge-equivalent, but as $S U(2)$ connections they are all distinct.

Finally we consider deformations of $S U(2)$ connections. Since $-1 \in \tilde{\Gamma}$ acts trivially on $l_{i}$ and $\beta^{j}$, our most general deformation is composed of even functions on $\widetilde{G}$. However, even functions on $\widetilde{G}$ are in $1-1$ correspondence with arbitrary functions on $\tilde{G} / Z_{2}=G$, so the classification of deformations of $S U(2)$ connections is identical to that of $S O(3)$ connections.

\section{Existence and Classification of Yang-Mills Solutions}

In this section we prove the existence of a family of equivariant Yang-Mills solutions, parametrized by $r$ and $t$. Specifically,

Theorem 3.1. For each pair of non-negative real numbers $(r, t)$ there exists a YangMills $(r, t)$-connection on $P_{X}$. Furthermore:

i. If $r$ and $t$ are both greater than 1 , or both strictly between 0 and 1 , then the solution has non-self-dual curvature.

ii. If $r \geqq 1 \geqq t$, or if $t=0$, then the solution has anti-self-dual curvature. If $t \geqq 1 \geqq r$, or if $r=0$, then the solution has self-dual curvature.

This, together with the general properties of $(r, t)$ connections shown in Sect. 2, gives Theorem 1.1. Two theorems were proven in [SS2] which, taken together, establish the case $r>1, t>1$.

Theorem 3.2. [SS2] For each pair of non-negative real numbers $(r, t)$ with $r \neq 1, t \neq 1$, there exists a Yang-Mills $(r, t)$-connection on $P_{X}$.

Theorem 3.3. [SS2] There do not exist any finite-action self-dual $(r, t)$-connections with $r>1$. There do not exist any finite-action anti-self-dual $(r, t)$-connections with $t>1$.

To complete part (i) of Theorem 3.1, we must prove an analog of Theorem 3.3 for $0<r<1$ and $0<t<1$. This is done in Sect. 3.1. To prove part (ii) of Theorem 3.1, we construct solutions to the (anti-) self-duality equations near $\theta=0$ and show that, for appropriate values of $r$ and $t$, these solutions can be extended to $\theta=\pi / 3$. This is the content of Sect. 3.2. 
3.1 Non-self-dual Yang-Mills connections: $0<r, t<1$.

Theorem 3.4. There do not exist any finite-action anti-self-dual $(r, t)$-connections with $r<1$ and $t \neq 0$. There do not exist any finite-action self-dual $(r, t)$-connections with $t<1$ and $r \neq 0$.

Proof. We prove the first statement, the second being similar. Let $a$ be a finiteaction anti-self-dual $(r, t)$ connection with $0<r<1$. Then, by the finite action boundary conditions, $a_{1}(0)=a_{2}(0)=0$. Let $\varepsilon=(1-r) / 2$. Since $a_{3}$ is continuous, there is a neighborhood $N$ of $\theta=0$ where $0<a_{3}<1-\varepsilon$.

The function $a(\theta)$ satisfies the anti-self-duality (ASD) equations

$$
a_{1}^{\prime}=-\frac{\left(a_{1}+a_{2} a_{3}\right)}{G_{1}}, \quad a_{2}^{\prime}=-\frac{\left(a_{2}+a_{1} a_{3}\right)}{G_{2}}, \quad a_{3}^{\prime}=-\frac{\left(a_{3}+a_{1} a_{2}\right)}{G_{3}} .
$$

It is convenient to rewrite the first two of these equations as

$$
\left(a_{1} \pm a_{2}\right)^{\prime}=-\frac{1 \pm a_{3}}{2}\left(\frac{1}{G_{1}}+\frac{1}{G_{2}}\right)\left(a_{1} \pm a_{2}\right)-\frac{1 \mp a_{3}}{2}\left(\frac{1}{G_{1}}-\frac{1}{G_{2}}\right)\left(a_{1} \mp a_{2}\right) \text {. }
$$

If we let $T(\theta)=a_{1}(\theta)^{2}+a_{2}(\theta)^{2}$, then

$$
\begin{aligned}
T^{\prime}(\theta)= & -\left(G_{1}^{-1}+G_{2}^{-1}\right)\left(a_{1}^{2}+a_{2}^{2}+2 a_{1} a_{2} a_{3}\right)-\left(G_{1}^{-1}-G_{2}^{-1}\right)\left(a_{1}^{2}-a_{2}^{2}\right) \\
= & -\left(G_{1}^{-1}+G_{2}^{-1}\right) a_{3}\left(a_{1}+a_{2}\right)^{2}-\left(G_{1}^{-1}+G_{2}^{-1}\right)\left(1-a_{3}\right)\left(a_{1}^{2}+a_{2}^{2}\right) \\
& -\left(G_{1}^{-1}-G_{2}^{-1}\right)\left(a_{1}^{2}-a_{2}^{2}\right) .
\end{aligned}
$$

The first two terms on the second line are negative semi-definite on $N$. Moreover, since $\left(G_{1}^{-1}+G_{2}^{-1}\right)$ has a pole at $\theta=0$ while $\left(G_{1}^{-1}-G_{2}^{-1}\right)$ does not, and since $1-a_{3}>\varepsilon$ on $N$, the third term is dominated by the second on some smaller neighborhood $M$ of zero. Thus $T$ is non-increasing on $M$. However, $T(0)=0$, so $T$ must be exactly zero on $M$, so $a_{1}=a_{2}=0$ on $M$.

If $a_{1}=a_{2}=0$ at any point in $(0, \pi / 3)$, then they are zero on all of $(0, \pi / 3)$, as Eqs. (3.2) are linear in $a_{1,2}$. So $t=\lim _{\theta \rightarrow \pi / 3} a_{2}(\theta)=0$.

There is an anti-self-dual $(r, 0)$ solution for any $r$. It is

$$
a_{1}=a_{2}=0, \quad a_{3}(\theta)=r \exp \left(-\int_{0}^{\theta} \frac{d y}{G_{3}(y)}\right) .
$$

The integral of $1 / G_{3}$ diverges at $\theta=\pi / 3$, so $a_{3}(\pi / 3)=0$, as it should. There is also a self-dual $(0, t)$ solution for any $t$, namely

$$
a_{1}=a_{3}=0, \quad a_{2}(\theta)=t \exp \left(-\int_{\theta}^{\pi / 3} \frac{d y}{G_{2}(y)}\right) .
$$

\subsection{Anti-Self-Dual Yang-Mills Connections: $r \geqq 1 \geqq t$. In this section we prove}

Theorem 3.5. For each pair of real numbers $(r, t)$ with $r \geqq 1 \geqq t \geqq 0$, there exists a finite-energy solution to the reduced ASD equations with $a_{3}(0)=r$ and $a_{2}(\pi / 3)=t$. For each $t \geqq 1 \geqq r \geqq 0$ there exists a finite-energy solution to the reduced $\mathrm{SD}$ equations.

We prove only the first statement. The proof of the second is completely analogous. We begin with local analysis near $\theta=0$. 
Lemma 3.6. Suppose $r \geqq 1$. Then for any constant $c$ there exists a solution to the reduced ASD equations on a neighborhood of $\theta=0$, of the form

$$
\begin{aligned}
& a_{1}(\theta)=-c \theta^{(r-1) / 2}+O\left(\theta^{(r+1) / 2}\right), \\
& a_{2}(\theta)=c \theta^{(r-1) / 2}+O\left(\theta^{(r+1) / 2}\right), \\
& a_{3}(\theta)=r+O\left(\theta^{2}\right) .
\end{aligned}
$$

Proof. We solve the ASD equations by iteration. Let $\phi_{3}(\theta)=\exp \left(-\int_{0}^{\theta} \frac{d y}{G_{3}(y)}\right)$ and let $a_{1,2}=\phi_{1,2}^{1}(\theta)$, and $a_{1,2}=\phi_{1,2}^{2}(\theta)$ be solutions to the linear ODE system

$$
a_{1}^{\prime}=-\frac{\left(a_{1}+r a_{2}\right)}{G_{1}}, \quad a_{2}^{\prime}=-\frac{\left(a_{2}+r a_{1}\right)}{G_{2}} .
$$

We can choose our normalizations such that

$$
\begin{aligned}
& \phi_{1}^{1}(\theta)=-\theta^{(r-1) / 2}+O\left(\theta^{(r+1) / 2},\right. \\
& \phi_{2}^{1}(\theta)=\theta^{(r-1) / 2}+O\left(\theta^{(r+1) / 2}\right), \\
& \phi_{1}^{2}(\theta)=\theta^{(-r-1) / 2}+O\left(\theta^{(-r+1) / 2}\right), \\
& \phi_{2}^{2}(\theta)=\theta^{(-r-1) / 2}+O\left(\theta^{(-r+1) / 2}\right) .
\end{aligned}
$$

Let $a_{1,2}^{(0)}=c \phi_{1,2}^{1}, a_{3}^{(0)}=r \phi_{3}$, and let $a_{i}^{(k+1)}$ be solutions, of the form (3.6), to the differential equations

$$
\begin{aligned}
& a_{1}^{(k+1)^{\prime}}=-\left(a_{1}^{(k+1)}+r a_{2}^{(k+1)}\right) / G_{1}+\left[r-a_{3}^{(k)}\right] a_{2}^{(k)} / G_{1}, \\
& a_{2}^{(k+1)^{\prime}}=-\left(a_{2}^{(k+1)}+r a_{1}^{(k+1)}\right) / G_{2}+\left[r-a_{3}^{(k)}\right] a_{1}^{(k)} / G_{2}, \\
& a_{3}^{(k+1)^{\prime}}=-\left(a_{3}^{(k+1)}+a_{1}^{(k)} a_{2}^{(k)}\right) / G_{3} .
\end{aligned}
$$

These solutions can be written explicitly, using the method of variation of parameters:

$$
\begin{aligned}
& a_{1}^{(k+1)}(\theta)=\left(c+h_{1}^{(k)}(\theta)\right) \phi_{1}^{1}(\theta)+h_{2}^{(k)}(\theta) \phi_{1}^{2}(\theta), \\
& a_{2}^{(k+1)}(\theta)=\left(c+h_{1}^{(k)}(\theta)\right) \phi_{2}^{1}(\theta)+h_{2}^{(k)}(\theta) \phi_{2}^{2}(\theta), \\
& a_{3}^{(k+1)}(\theta)=\left(r+h_{3}^{(k)}(\theta)\right) \phi_{3}(\theta),
\end{aligned}
$$

where

$$
\begin{aligned}
& h_{1}^{(k)}(\theta)=\int_{0}^{\theta} W(y)\left(r-a_{3}^{(k)}(y)\right)\left[\frac{a_{2}^{(k)}(y) \phi_{2}^{2}(y)}{G_{1}(y)}-\frac{a_{1}^{(k)}(y) \phi_{1}^{2}(y)}{G_{2}(y)}\right] d y, \\
& h_{2}^{(k)}(\theta)=\int_{0}^{\theta} W(y)\left(r-a_{3}^{(k)}(y)\right)\left[\frac{-a_{2}^{(k)}(y) \phi_{2}^{1}(y)}{G_{1}(y)}+\frac{a_{1}^{(k)}(y) \phi_{1}^{1}(y)}{G_{2}(y)}\right] d y, \\
& h_{3}^{(k)}(\theta)=-\int_{0}^{\theta} \frac{a_{1}^{(k)}(y) a_{2}^{(k)}(y)}{\phi_{3}(y) G_{3}(y)} d y,
\end{aligned}
$$

and $W(y)=1 /\left(\phi_{1}^{1}(y) \phi_{2}^{2}(y)-\phi_{1}^{2}(y) \phi_{2}^{1}(y)\right)=-\theta / 2+O\left(\theta^{2}\right)$. For sufficiently small $\theta$, $a^{(k+1)}-a^{(k)}$ is (pointwise) small compared to $a^{(k)}-a^{(k-1)}$, so the iteration converges. Letting $a_{i}=\lim _{k \rightarrow \infty} a_{i}^{(k)}$ we get our desired solution, of form (3.6), to the ASD equations (3.1). 
Note that, if $c$ is positive, then for $\theta$ sufficiently small $a_{3}(\theta)$ and $a_{2}(\theta)$ are positive and $a_{1}(\theta)$ is negative. We next prove that these signs persist for all $\theta$.

Lemma 3.7. Suppose $a(\theta)$ is a solution of the reduced ASD equations, and that at some point $\theta_{0} \in(0, \pi / 3), a_{2,3}\left(\theta_{0}\right)>0>a_{1}\left(\theta_{0}\right)$. Then for all $\theta \in\left(\theta_{0}, \pi / 3\right), a_{2,3}(\theta)>$ $0>a_{1}(\theta)$.

Proof. Suppose the conclusion is false. Then there is a smallest value of $\theta$, call it $\theta_{1}$, where one (or more) of the $a_{i}$ 's is equal to zero. If two or three of the functions are zero at $\theta_{1}$, then the ASD equations imply that they are zero for all $\theta$, and in particular at $\theta_{0}$, contradicting the assumption. So all we need rule out is the possibility that exactly one of the $a_{i}$ 's is zero at $\theta_{1}$.

Suppose $a_{3}\left(\theta_{1}\right)=0$ (the other two cases are similar). Since $a_{3}(\theta)$ is positive for all $\theta \in\left(\theta_{0}, \theta_{1}\right), a_{3}^{\prime}\left(\theta_{1}\right)$ cannot be positive. However, $a_{3}^{\prime}\left(\theta_{1}\right)=$ $-a_{1}\left(\theta_{1}\right) a_{2}\left(\theta_{1}\right) / G_{3}\left(\theta_{1}\right)$ is positive. Contradiction.

Combining Lemmas 3.6 and 3.7, we see that there is a 1-parameter family of solutions to the ASD equations, with definite sign properties. However, it is not immediately clear whether a given solution is defined on all of $[0, \pi / 3]$ or just on a neighborhood of zero, and whether it has finite or infinite energy. The finite vs. infinite energy question is resolved by the following lemmas.

Lemma 3.8. Suppose $a(\theta)$ is a solution of the reduced ASD equations with $r>0$, and $\operatorname{CS}(\theta)$ is the reduced Chern-Simons functional. The action $S(a)$, restricted to any subinterval $\left(\theta_{0}, \theta_{1}\right) \subset(0, \pi / 3)$ is $8 \pi^{2}\left(\operatorname{CS}\left(\theta_{1}\right)-\operatorname{CS}\left(\theta_{0}\right)\right)$. Furthermore, either

1. $a$ is a finite-action reduced connection defined on all of $[0, \pi / 3], \operatorname{CS}(\theta)$ is positive for all $\theta \in(0, \pi / 3)$, and $S(a) \leqq \pi^{2} r^{2}$, or

2. There is a point $\theta_{0} \in(0, \pi / 3)$ such that $\operatorname{CS}\left(\theta_{0}\right)=0$, and a has infinite action. (a may or may not be defined on all of $[0, \pi / 3])$.

Proof. If the curvature $F_{A}$ is anti-self-dual, then the Chern-Weil form equals $1 / 8 \pi^{2}$ times the action density. Integrating the Chern-Weil form first over the symmetry group and then over $\left(\theta_{0}, \theta_{1}\right)$ we get $\operatorname{CS}\left(\theta_{1}\right)-\operatorname{CS}\left(\theta_{0}\right)$. Integrating the action density we get the action.

1) Now suppose $a$ is a finite-action ASD connection. We first show that $a$ is defined on all of $[0, \pi / 3]$. We already know that $a$ is defined on some neighborhood $[0, \delta]$. Since $G_{3}$ is bounded below on $[\delta, \pi / 6]$, finite action implies that $a_{3}$ is of finite variation on $[\delta, \pi / 6]$ (see [SS2] for the precise estimates), and in particular is bounded. Given bounded $a_{3}$, the equations for $a_{1,2}$ are linear with bounded coefficients, and so $a_{1,2}$ cannot blow up on $[\delta, \pi / 6]$. Similarly, $G_{2}$ is bounded below on $[\pi / 6, \pi / 3]$, so $a_{2}$ is bounded, so the equations for $a_{1,3}$ cannot give blowup at any point prior to $\pi / 3$. Thus $a$ is defined on all of $[0, \pi / 3)$. The finite-action boundary conditions then give finite limits at $\pi / 3$.

The finite-action boundary conditions at $\pi / 3$ also imply that $\operatorname{CS}(\pi / 3)=t^{2} \geqq 0$. For any point $\theta_{0} \in(0, \pi / 3), \quad \operatorname{CS}\left(\theta_{0}\right)=t^{2}+($ action on $(\theta, \pi / 3)) / 8 \pi^{2}>0$. $S(a)=8 \pi^{2}(\operatorname{CS}(0)-\operatorname{CS}(\pi / 3))=\pi^{2}\left(r^{2}-t^{2}\right) \leqq \pi^{2} r^{2}$.

2) If the action is unbounded, there is a point $\theta_{0}$ where the action on $\left[0, \theta_{0}\right]$ equals $\pi^{2} r^{2}$. Since $\operatorname{CS}(0)=r^{2} / 8, \operatorname{CS}\left(\theta_{0}\right)$ must equal zero. 
Note that $\operatorname{CS}(\theta)$ is a non-increasing function of $\theta$. If we can show that $\operatorname{CS}(\theta)$ is positive in a neighborhood of $\pi / 3$, then it must be positive everywhere and $a$ has finite action.

Lemma 3.9. If $0<a_{2}(\theta) \leqq 1$, then $\operatorname{CS}(\theta)>0$.

Proof.

$$
\begin{aligned}
\operatorname{CS}(\theta) & =\left(a_{2}^{2}+a_{1}^{2}+a_{3}^{2}+2 a_{1} a_{2} a_{3}\right) / 8 \\
& =\left(a_{2}^{2}+a_{2}\left(a_{1}+a_{3}\right)^{2}+\left(1-a_{2}\right)\left(a_{1}^{2}+a_{3}^{2}\right)\right) / 8 .
\end{aligned}
$$

As long as $0<a_{2} \leqq 1$, the first term is positive definite and the remaining terms are positive semi-definite, so the sum is positive.

If we can maintain $a_{2} \leqq 1$ in a neighborhood of $\pi / 3$, then we can prove that $a$ is a finite-action solution. The key estimate is the following:

\section{Lemma 3.10.}

1. Suppose $0 \leqq \theta_{1} \leqq \theta_{2} \leqq \delta \leqq 1 / 10$. If the energy between $\theta_{1}$ and $\theta_{2}$ is bounded by a constant $\bar{\pi}^{2} M^{2}$, then $\left|a_{3}\left(\theta_{1}\right)-a_{3}\left(\theta_{2}\right)\right| \leqq 2 M \delta$. If the energy between 0 and $\theta_{2}$ is bounded by $\pi^{2} M^{2}$, then $\left|r-a_{3}\left(\theta_{2}\right)\right| \leqq 2 M \theta_{2}$.

2. Suppose $\pi / 3-1 / 10 \leqq \pi / 3-\delta \leqq \theta_{1} \leqq \theta_{2} \leqq \pi / 3$. If the energy between $\theta_{1}$ and $\theta_{2}$ is bounded by a constant $\pi^{2} M^{2}$, then $\left|a_{2}\left(\theta_{1}\right)-a_{2}\left(\theta_{2}\right)\right| \leqq 2 M \delta$. If the energy between $\theta_{1}$ and $\pi / 3$ is bounded by $\pi^{2} M^{2}$, then $\left|t-a_{2}\left(\theta_{1}\right)\right| \leqq 2 M\left(\pi / 3-\theta_{1}\right)$.

Proof. (1) $G_{3}$ is a decreasing function, and for $y<1 / 10$ we have $G_{3}(y)>1 /(4 y)$. As a result,

$$
\begin{aligned}
\left(a_{3}\left(\theta_{1}\right)-a_{3}\left(\theta_{2}\right)\right)^{2} & \leqq \frac{\delta}{\theta_{2}-\theta_{1}}\left(a_{3}\left(\theta_{1}\right)-a_{3}\left(\theta_{2}\right)\right)^{2} \leqq \delta \int_{\theta_{1}}^{\theta_{2}}\left(a_{3}^{\prime}(y)\right)^{2} d y \\
& \leqq 4 \delta^{2} \int_{\theta_{1}}^{\theta_{2}} G_{3}(y)\left(a_{3}^{\prime}(y)\right)^{2} d y \leqq 4 M^{2} \delta^{2} .
\end{aligned}
$$

The bound on $\left|r-a_{3}\left(\theta_{2}\right)\right|$ then follows from taking $\theta_{1}=0$ and $\delta=\theta_{2}$. The proof of statement 2 is similar, as $G_{2}(y)=G_{3}(\pi / 3-y)$.

Proposition 3.11. Given $r>0$ and $\varepsilon \in(0,1)$, there exists $a \delta$ such that

1. Every ASD reduced connection a defined on $[0, \pi / 3-\delta]$ with $a_{3}(0)=r$ and $0<a_{2}(\pi / 3-\delta)<1-\varepsilon$ can be extended to be a finite-action ASD connection on all of $[0, \pi / 3]$ with $\left|a_{2}(\pi / 3)-a_{2}(\pi / 3-\delta)\right|<\varepsilon$.

2. No ASD reduced connection defined on $[0, \pi / 3-\delta]$ with $a_{3}(0)=r$ and with $a_{2}(\pi / 3-\delta)>1+\varepsilon$ can be extended to a finite-action ASD solution on $[0, \pi / 3]$.

Proof. Let $\delta=\min (1 / 10, \varepsilon /(2 r))$.

1) Suppose $0<a_{2}(\pi / 3-\delta)<1-\varepsilon$ and $a$ cannot be extended to a finite-action ASD connection on all of $[0, \pi / 3]$. Then by Lemma 3.8 there must exist a first point $\theta_{2}$ where $\operatorname{CS}\left(\theta_{2}\right)=0$. Let $\theta_{1}=\pi / 3-\delta$. By Lemma 3.9 $\operatorname{CS}\left(\theta_{1}\right)>0$, so $\theta_{2}>\theta_{1}$. Also by Lemma 3.9, $a_{2}\left(\theta_{2}\right)>1$, so $\left|a_{2}\left(\theta_{2}\right)-a_{2}\left(\theta_{1}\right)\right|>\varepsilon$. However, the energy between $\theta_{1}$ and $\theta_{2}$ cannot be greater than the total energy between 0 and $\theta_{2}$, which by Lemma 3.8 is $\pi^{2} r^{2}$. So by Lemma $3.10,\left|a_{2}\left(\theta_{2}\right)-a_{2}\left(\theta_{1}\right)\right| \leqq \varepsilon$, which is a contradiction. The bound on $a_{2}(\pi / 3)$ also follows from Lemma 3.10. 
2) Now suppose $a_{2}(\pi / 3-\delta)>1+\varepsilon$. If $a$ can be extended to a finite-action solution on $[0, \pi / 3]$, then the energy between $\pi / 3-\delta$ and $\pi / 3$ must be less than $\pi^{2} r^{2}$ (by Lemma 3.8). By Lemma 3.10, $t \geqq a_{2}(\pi / 3-\delta)-\varepsilon>1$. But by Theorem 3.3 there are no finite-action ASD connections with $t>1$.

Proposition 3.11 gives a fairly coherent description of the family of ASD solutions given by Lemma 3.6. For $c=0$ we get the explicit solution of Eq. (3.4). As $c$ increases, $a_{2}(\theta)$ increases for any fixed $\theta$, and in particular $t$ increases. As long as $t$ remains less than 1 , the solution has finite energy. Eventually $c$ hits a critical value, which we call $c_{1}$, for which $t=1$. For $c$ slightly greater than $c_{1}$ we get infinite energy solutions on $(0, \pi / 3)$. The limit $t=a_{2}(\pi / 3)$ still exists (and is greater than 1 ), but $a_{1}$ and $a_{3}$ diverge as $\theta$ approaches $\pi / 3$. Eventually $c$ reaches another critical value, which we call $c_{2}$, after which the solution blows up prior to $\pi / 3$. To complete the proof of Theorem 3.5 we must show that $c_{1}$ is neither zero nor infinite, and that, for $c \in\left[0, c_{1}\right], t$ varies continuously with $c$.

The critical value $c_{2}$ is not needed for the proof of Theorem 3.5. We merely remark, without proof, that it corresponds to $t=2$.

Lemma 3.12. Let $r \geqq 1$ be fixed and let $a^{c}$ denote the ASD solution of Lemma 3.6 with constant $c$. Let $c_{1}=\inf \left\{c \mid a^{c}\right.$ has infinite action $\}$. Then

1. If $c_{1} \in(0, \infty)$, then $a^{c_{1}}$ is a finite action connection with $t=1$.

2. The boundary value $t$ is a continuous function of $c$ for $c \in\left[0, c_{1}\right]$.

3. $c_{1} \in(0, \infty)$.

Proof. 1) For small $\theta$, it is clear from Eq. (3.6) that $a^{c}(\theta)$ depends continuously on $c$. Since solutions to ODE's depend continuously on their initial conditions, $a^{c}(\theta)$ must depend continuously on $c$ for any $\theta \in(0, \pi / 3)$ such that $a^{c}(\theta)$ is defined.

Let $S_{\delta}(a)$ be the energy of the reduced connection $a$ between $\theta=\delta$ and $\theta=\pi / 3-\delta$. For ASD connections, the derivative and non-derivative terms are equal, so

$$
S_{\delta}(a)=2 \pi^{2} \int_{\delta}^{\pi / 3}\left[\frac{\left(a_{1}+a_{2} a_{3}\right)^{2}}{G_{1}}+\frac{\left(a_{2}+a_{1} a_{3}\right)^{2}}{G_{2}}+\frac{\left(a_{3}+a_{1} a_{2}\right)^{2}}{G_{3}}\right] .
$$

As $c \rightarrow c_{1}$ from below, $a^{c}$ approaches $a^{c_{1}}$ pointwise. Furthermore, all of the $a^{c}$ s with $c<c_{1}$ have energies bounded by $\pi^{2} r^{2}$ (Lemma 3.8), so we can find a fixed bound for $a^{c}$ on $[\delta, \pi / 3-\delta]$, independent of $c$, as in the proof of Lemma 3.8. Since the integrand for $S_{\delta}\left(a^{c}\right)$ approaches that of $S_{\delta}\left(a^{c_{1}}\right)$ and is bounded, $S_{\delta}\left(a^{c}\right)$ approaches $S_{\delta}\left(a^{c_{1}}\right)$. But $S_{\delta}\left(a^{c}\right) \leqq S\left(a^{c}\right) \leqq \pi^{2} r^{2}$, so $S_{\delta}\left(a^{c_{1}}\right) \leqq \pi^{2} r^{2}$. Finally,

$$
S\left(a^{c_{1}}\right)=\lim _{\delta \rightarrow 0} S_{\delta}\left(a^{c_{1}}\right) \leqq \pi^{2} r^{2}<\infty .
$$

Since $a^{c_{1}}$ has finite action, $t=a_{2}^{c_{1}}(\pi / 3)$ must exist. It is non-negative (Lemma 3.7) and cannot be greater than 1 (Theorem 3.3). We next show that $t$ cannot be less than 1 .

Suppose $t<1$. Let $\varepsilon=(1-t) / 3$, and let $\delta=\min (1 / 10, \varepsilon /(2 r))$. Then, by Proposition 3.11, $\left|t-a_{2}^{c_{1}}(\pi / 3-\delta)\right|<\varepsilon$, so $a_{2}^{c_{1}}(\pi / 3-\delta)<1-2 \varepsilon$. But then for all $c$ sufficiently close to $c_{1}, a_{2}^{c}(\pi / 3-\delta)<1-\varepsilon$, so by Proposition 3.11 all such $a^{c}$ 's have finite action. This contradicts the definition of $c_{1}$.

2) By Lemma 3.10, the family $a_{2}^{c}$ is uniformly equicontinuous near $\theta=\pi / 3$ for $c \leqq c_{1}$. This, combined with the continuous dependence of $a_{2}^{c}(\theta)$ on $c$ for fixed $\theta<\pi / 3$, gives continuous dependence of $t=a_{2}^{c}(\pi / 3)$ on $c$. 
3) To show that $c_{1}>0$ we pick $\varepsilon=1 / 2$ and let $\delta=\min (1 / 10, \varepsilon /(2 r))$. Since $a_{2}^{0}(\pi / 3-\delta)=0$, for all sufficiently small $c$ we have $a_{2}^{c}(\pi / 3-\delta)<1-\varepsilon$, so $a^{c}$ has finite action. Thus $c_{1}>0$.

To show that $c_{1}<\infty$, we show that for sufficiently large $c, a^{c}$ has action greater than $\pi^{2} r^{2}$, and so by Lemma 3.8 has infinite action. We do this by choosing a $c$ so big that either there is energy greater than $\pi^{2} r^{2}$ in a small interval $(0, \delta)$, or $a_{2}(\delta)$ is so big that it takes energy greater than $\pi^{2} r^{2}$ on $(\delta, \pi / 3)$ to bring $a_{2}$ back down.

First we bound the variation of $a_{3}$. By Lemma 3.10, if the action between 0 and $\theta$ is bounded by $\pi^{2} r^{2}$, then

$$
\left|a_{3}(\theta)-r\right| \leqq 2 r \theta
$$

Next we look at the growth of $\left|a_{1}-a_{2}\right|$. By Eq. (3.2),

$$
\begin{aligned}
d \log \left|a_{1}-a_{2}\right| / d \theta= & \left(a_{3}-1\right)\left(G_{1}^{-1}+G_{2}^{-1}\right) / 2-\left(a_{3}+1\right) \\
& \times\left(G_{1}^{-1}-G_{2}^{-1}\right)\left(a_{1}+a_{2}\right) /\left[2\left(a_{1}-a_{2}\right)\right] \\
\geqq & \left(a_{3}-1\right)\left(G_{1}^{-1}+G_{2}^{-1}\right) / 2-\left(a_{3}+1\right)\left|G_{1}^{-1}-G_{2}^{-1}\right| / 2,
\end{aligned}
$$

where we have used the fact that $a_{1}$ and $a_{2}$ have different signs (Lemma 3.7), so $\left|a_{1}-a_{2}\right|>\left|a_{1}+a_{2}\right|$. Comparing this to the growth of $\theta^{(r-1) / 2}$ we see that

$$
\begin{aligned}
\log \left(\frac{\left|a_{1}-a_{2}\right|}{2 c \theta^{(r-1) / 2}}\right) \geqq & \int_{0}^{\theta} d y\left(a_{3}(y)-r\right)\left(G_{1}^{-1}(y)+G_{2}^{-1}(y)\right) / 2+(r-1)\left(G_{1}^{-1}(y)\right. \\
& \left.+G_{2}^{-1}(y)-y^{-1}\right) / 2-\left(a_{3}(y)+1\right)\left|G_{1}^{-1}-G_{2}^{-1}\right| / 2 .
\end{aligned}
$$

The integrals on the right-hand side can all be bounded, independent of $c$, using (3.16). As a result, for any small $\delta$, by choosing $c$ large enough we can force $\left|a_{1}(\delta)-a_{2}(\delta)\right|$ to be as large as we wish. If we can prove the bound $\left|a_{1}(\delta)+a_{2}(\delta)\right|$ $<\left|a_{1}(\delta)-a_{2}(\delta)\right| / 2$, independently of $c$, then we will have forced $a_{2}(\delta)$ to be arbitrarily large, and we will be done.

However, by Eqs. (3.2), $\left|a_{1}+a_{2}\right|^{\prime} \leqq\left|\left(a_{3}-1\right)\left(G_{1}^{-1}-G_{2}^{-1}\right)\left(a_{1}-a_{2}\right)\right| / 2$. Integrating this from 0 to $\theta$, using (3.16) and the fact that $\left|a_{1}-a_{2}\right|$ is an increasing function, gives our desired bound on $\left|a_{1}+a_{2}\right|$.

Proof of Theorem 3.5: For any fixed $r \geqq 1$, consider the family of functions $a^{c}$ with $c \in\left[0, c_{1}\right]$. These are all finite-energy solutions to the ASD equations. Since $t$ depends continuously on $c, c=0$ implies $t=0$, and $c=c_{1}$ implies $t=1$. Therefore as $c$ increases from 0 to $c_{1}, t$ must take on all values between 0 and 1 .

Theorems 3.2, 3.3, 3.4, and 3.5, together with the explicit solutions of Eqs. (3.4) and (3.5), cover all the cases of Theorem 3.1.

\section{Anti-self-dual Connections}

In this section we take $r \geqq 1 \geqq t$ and look at deformations of the anti-self-dual YM solutions of Theorem 3.5. The goal is to prove Theorems 1.2 and 1.3, which give the 
formal dimensions of the moduli spaces of anti-self-dual connections with appropriate boundary conditions. These proofs appear at the end of Sect. 4.4.

In Sect. 4.1 we derive the linearized (anti)self-duality equations for deformations of the connection. These form a countable collection of linear ODE systems, one for each representation of the symmetry group $S O(3)$. We also write down an appropriate gauge condition. Finally, we compute the second variation of the Yang-Mills functional (i.e. the Hessian).

In Sect. 4.2 we derive appropriate boundary conditions for the ODEs of Sect. 4.1. The boundary conditions differ, depending on whether $r$ is an odd integer or not. When $r$ is an odd integer the boundary conditions at $\theta=0$ follow from smoothness on $S^{4}$. When $r$ is not an odd integer we have a choice of boundary conditions, depending on how we define our space of connections and our gauge group. We consider both the strongest reasonable boundary conditions, which we term "regularity," and the weakest reasonable conditions, which we term "weak regularity."

In Sect. 4.3 we find approximate solutions to the linearized ASD equations, and show that these solutions have the same asymptotic behavior near $\theta=0$ as the exact solutions.

Finally, in Sect. 4.4 we compute the dimension of the moduli space. For each representation of $S O(3)$, we compute the dimension of the stable manifold of the ASD equations near $\theta=0$ and $\theta=\pi / 3$. For generic metrics these manifolds intersect transversally, so we can compute the dimension of the space of admissible solutions to the ASD equations. Summing over all representations we get the dimension of the moduli space. We get two sets of answers, one for regular solutions and one for weakly regular solutions.

4.1 The ASD Equations. Let $a=\left(a_{1}, a_{2}, a_{3}\right)$ be a reduced $(r, t)$ connection, corresponding to an equivariant Yang-Mills connection $A$. We wish to consider deformations of $A$. As discussed in Sect. 2.4, these take the general form

$$
\delta A=\sum_{i, j} \alpha_{i j}(\theta, g) \beta^{i} \otimes l_{j}+\sum_{i} \gamma_{i}(\theta, g) d \theta \otimes l_{i} .
$$

One easily establishes the following four propositions. Propositions 4.1, 4.3, and 4.4 are direct computations, while Proposition 4.2 follows from Proposition 4.1 and Eqs. (2.6).

Proposition 4.1. The first variation of the curvature is given by

$$
\begin{aligned}
\delta F= & d_{A}(\delta A) \\
= & \left(\alpha_{11}^{\prime}-l_{1} \gamma_{1}\right)(011)+\left(\alpha_{12}^{\prime}-l_{1} \gamma_{2}-a_{1} \gamma_{3}\right)(012)+\left(\alpha_{13}^{\prime}-l_{1} \gamma_{3}+a_{1} \gamma_{2}\right)(013) \\
& +\left(l_{2} \alpha_{31}-l_{3} \alpha_{21}-\alpha_{11}-a_{3} \alpha_{22}-a_{2} \alpha_{33}\right)(231) \\
& +\left(l_{2} \alpha_{32}-l_{3} \alpha_{22}-\alpha_{12}+a_{3} \alpha_{21}\right)(232) \\
& +\left(l_{2} \alpha_{33}-l_{3} \alpha_{23}-\alpha_{13}+a_{2} \alpha_{31}\right)(233)+\text { cyclic },
\end{aligned}
$$

where $(0 j k)$ and $(i j k)$ are shorthand for $d \theta \wedge \beta^{j} \otimes l_{k}$ and $\beta^{i} \wedge \beta^{j} \otimes l_{k}$, respectively, and ' denotes $\partial / \partial \theta$. 
Proposition 4.2. The linearized anti-self-duality equations $* \delta F=-\delta F$ are

$$
\begin{aligned}
G_{1}\left(\alpha_{11}^{\prime}-l_{1} \gamma_{1}\right) & =\left(l_{2} \alpha_{31}-l_{3} \alpha_{21}-\alpha_{11}-a_{3} \alpha_{22}-a_{2} \alpha_{33}\right), \\
G_{1}\left(\alpha_{12}^{\prime}-l_{1} \gamma_{2}-a_{1} \gamma_{3}\right) & =\left(l_{2} \alpha_{32}-l_{3} \alpha_{22}-\alpha_{12}+a_{3} \alpha_{21}\right), \\
G_{1}\left(\alpha_{13}^{\prime}-l_{1} \gamma_{3}+a_{1} \gamma_{2}\right) & =\left(l_{2} \alpha_{33}-l_{3} \alpha_{23}-\alpha_{13}+a_{2} \alpha_{31}\right), \\
G_{2}\left(\alpha_{21}^{\prime}-l_{2} \gamma_{1}+a_{2} \gamma_{3}\right) & =\left(l_{3} \alpha_{11}-l_{1} \alpha_{31}-\alpha_{21}+a_{3} \alpha_{12}\right), \\
G_{2}\left(\alpha_{22}^{\prime}-l_{2} \gamma_{2}\right) & =\left(l_{3} \alpha_{12}-l_{1} \alpha_{32}-\alpha_{22}-a_{1} \alpha_{33}-a_{3} \alpha_{11}\right), \\
G_{2}\left(\alpha_{23}^{\prime}-l_{2} \gamma_{3}-a_{2} \gamma_{1}\right) & =\left(l_{3} \alpha_{13}-l_{1} \alpha_{33}-\alpha_{23}+a_{1} \alpha_{32}\right), \\
G_{3}\left(\alpha_{31}^{\prime}-l_{3} \gamma_{1}-a_{3} \gamma_{2}\right) & =\left(l_{1} \alpha_{12}-l_{2} \alpha_{11}-\alpha_{31}+a_{2} \alpha_{13}\right), \\
G_{3}\left(\alpha_{32}^{\prime}-l_{3} \gamma_{2}+a_{3} \gamma_{1}\right) & =\left(l_{1} \alpha_{22}-l_{2} \alpha_{12}-\alpha_{32}+a_{1} \alpha_{23}\right), \\
G_{3}\left(\alpha_{33}^{\prime}-l_{3} \gamma_{3}\right) & =\left(l_{1} \alpha_{23}-l_{2} \alpha_{13}-\alpha_{33}-a_{1} \alpha_{22}-a_{2} \alpha_{11}\right) .
\end{aligned}
$$

Proposition 4.3. The covariant divergence of $\delta A$ is

$$
\begin{aligned}
d_{A}^{*}(\delta A)= & {\left[\frac{l_{1} \alpha_{11}}{f_{1}^{2}}+\frac{l_{2} \alpha_{21}-a_{2} \alpha_{23}}{f_{2}^{2}}+\frac{l_{3} \alpha_{31}+a_{3} \alpha_{32}}{f_{3}^{2}}+\frac{\left(f_{1} f_{2} f_{3} \gamma_{1}\right)^{\prime}}{f_{1} f_{2} f_{3}}\right] l_{1} } \\
& +\left[\frac{l_{1} \alpha_{12}+a_{1} \alpha_{13}}{f_{1}^{2}}+\frac{l_{2} \alpha_{22}}{f_{2}^{2}}+\frac{l_{3} \alpha_{32}-a_{3} \alpha_{31}}{f_{3}^{2}}+\frac{\left(f_{1} f_{2} f_{3} \gamma_{2}\right)^{\prime}}{f_{1} f_{2} f_{3}}\right] l_{2} \\
& +\left[\frac{l_{1} \alpha_{13}-a_{1} \alpha_{12}}{f_{1}^{2}}+\frac{l_{2} \alpha_{23}-a_{2} \alpha_{21}}{f_{2}^{2}}+\frac{l_{3} \alpha_{33}}{f_{3}^{2}}+\frac{\left(f_{1} f_{2} f_{3} \gamma_{3}\right)^{\prime}}{f_{1} f_{2} f_{3}}\right] l_{3} .
\end{aligned}
$$

Proposition 4.4. The Hessian is given by

$$
\delta^{2} S=\langle\delta F, \delta F\rangle+2\left\langle F, \delta^{2} F\right\rangle,
$$

where the second variation of the curvature is

$$
\begin{aligned}
\delta^{2} F= & {[\delta A, \delta A] } \\
= & \left(\gamma_{2} \alpha_{13}-\gamma_{3} \alpha_{12}\right)(011)+\left(\gamma_{3} \alpha_{11}-\gamma_{1} \alpha_{13}\right)(012)+\left(\gamma_{1} \alpha_{12}-\gamma_{2} \alpha_{11}\right)(013) \\
& +\left(\alpha_{12} \alpha_{23}-\alpha_{22} \alpha_{13}\right)(121)+\left(\alpha_{13} \alpha_{21}-\alpha_{23} \alpha_{11}\right)(122)+\left(\alpha_{11} \alpha_{22}-\alpha_{21} \alpha_{12}\right)(12) \\
& + \text { cyclic. }
\end{aligned}
$$

4.2 Boundary Values. We wish to study the linearized anti-self-duality equations (4.3), together with the gauge-fixing condition $d_{A}^{*}(\delta A)=0$. We restrict our attention to a particular representation of $S O(3)$, and decompose the functions $\alpha_{i j}(\theta, g)$ and $\gamma_{i}(\theta, g)$ along the basis of Proposition 2.1. That is, we write

$$
\alpha_{13}(\theta, g)=\sum_{m>0, \text { odd }} \alpha_{13}^{m}(\theta) \Psi_{l, m}^{-}(g),
$$

with similar expansions for the other components of $\alpha$ and $\gamma$. The ASD and gauge-fixing equations then become a system of ODEs for the functions $\alpha_{i j}^{m}$ and $\gamma_{i}^{m}$ on the interval $[0, \pi / 3]$. The question is what boundary conditions to impose at 0 and $\pi / 3$. The answer depends on $r$ and $t$. 
Proposition 4.5. Let $r$ be a positive odd integer, let $A$ be an equivariant connection that is smooth on a neighborhood of $Q_{0}$, and let $\delta A$ be a deformation of $A$ that is smooth near $Q_{0}$. Then, with the exception of the 6 modes listed below, all components of $\alpha$ and $\gamma$ are zero at $\theta=0$, and $\alpha_{3 j}^{m \prime}(0)=0$ for all $j, m$.

If $r \geqq 3$, then the 6 exceptional modes are as follows:

1. $\alpha_{23}^{1}(0)=-\alpha_{13}^{1}(0)$ may be nonzero.

2. $\alpha_{33}^{2 \prime}(0)=2 \gamma_{3}^{2}(0)$ may be nonzero.

3. $-\alpha_{22}^{r-1}(0)=\alpha_{11}^{r-1}(0)=\alpha_{12}^{r-1}(0)=\alpha_{21}^{r-1}(0)$ may be nonzero.

4. $\alpha_{22}^{r+1}(0)=\alpha_{11}^{r+1}(0)=-\alpha_{21}^{r+1}(0)=\alpha_{12}^{r+1}(0)$ may be nonzero.

5. $\alpha_{31}^{r-2 \prime}(0)=\alpha_{32}^{r-2 \prime}(0)=2 \gamma_{2}^{r-2}(0)=-2 \gamma_{1}^{r-2}(0)$ may be nonzero.

6. $\alpha_{31}^{r+2 \prime}(0)=\alpha_{32}^{r+2 \prime}(0)=2 \gamma_{1}^{r+2}(0)=-2 \gamma_{2}^{r+2}(0)$ may be nonzero.

If $r=1$, then the exceptional modes 1, 2, 4 and 6 are as before. However, in place of modes 3 and 5 we have

3.' If $l$ is odd then $\alpha_{12}^{0}(0)=\alpha_{21}^{0}(0)$ may be nonzero.

If $l$ is even then $\alpha_{11}^{0}(0)=-\alpha_{22}^{0}(0)$ may be nonzero.

5.' $\alpha_{31}^{1 \prime}(0)=-\alpha_{32}^{1 \prime}(0)=2 \gamma_{2}^{1}(0)=2 \gamma_{1}^{1}(0)$ may be nonzero.

Note that if $r>l-2$, then some of the exceptional modes may not exist, as $m$ cannot be greater than $l$.

Proof. We choose appropriate nonsingular coordinates near $Q_{0}$ and write down an arbitrary smooth connection form $\delta A$ in these coordinates, relative to the section $\delta$ of formula (2.16). We then write $\delta A_{\delta}$ a different way, by starting with the expansion (4.1), applying the transition function (2.15) and doing a change of coordinates. Comparing the two expressions shows that the only possible nonzero coefficients at zero are those of modes 1-6.

An arbitrary element $Q \in S^{4} \subset V$ can be written in terms of the basis (2.2), as

$$
Q=\sum_{i=0}^{4} x_{i} Q_{i} .
$$

We use $\left(x_{1}, x_{2}, x_{3}, x_{4}\right)$ as coordinates for $S^{4}$ near $Q_{0}$. Our connection form near $Q_{0}$, relative to the section $\delta$ is then

$$
\delta A_{\delta}=\sum_{i=1}^{4} \sum_{j=1}^{3} \delta A_{i}^{j} d x_{i} \otimes l_{j} .
$$

Converting between the $\{x\}$ and $\{\theta, y\}$ coordinates is easy. We apply the group element $g=\exp \left(y_{1} K_{1}+y_{2} K_{2}\right) \exp \left(y_{3} K_{3}\right)$ to $Q_{\theta}$, and decompose relative to the basis (4.8). To first order in $y_{1}, y_{2}, \theta$ we have

$$
\left(x_{1}, x_{2}, x_{3}, x_{4}\right)=\left(y_{2} \sqrt{3} / 2,-y_{1} \sqrt{3} / 2, \theta \cos \left(2 y_{3}\right), \theta \sin \left(2 y_{3}\right)\right) .
$$


Our standard vector fields on $S^{4}$ are, to lowest order, given by

$$
\begin{aligned}
& \partial_{\theta}=\cos \left(2 y_{3}\right) \partial / \partial x_{3}+\sin \left(2 y_{3}\right) \partial / \partial x_{4}, \\
& l_{1}=\operatorname{ad}_{g}\left(K_{1}\right)\left(Q_{\theta}\right)=\sqrt{3} \sin \left(y_{3}\right) \partial / \partial x_{1}-\sqrt{3} \cos \left(y_{3}\right) \partial / \partial x_{2}, \\
& l_{2}=\operatorname{ad}_{g}\left(K_{2}\right)\left(Q_{\theta}\right)=\sqrt{3} \cos \left(y_{3}\right) \partial / \partial x_{1}+\sqrt{3} \sin \left(y_{3}\right) \partial / \partial x_{2}, \\
& l_{3}=\partial / \partial y_{3}=2 \theta\left(-\sin \left(2 y_{3}\right) \partial / \partial x_{3}+\cos \left(2 y_{3}\right) \partial / \partial x_{4}\right),
\end{aligned}
$$

and so their duals are

$$
\begin{aligned}
& d \theta=\cos \left(2 y_{3}\right) d x_{3}+\sin \left(2 y_{3}\right) d x_{4}, \\
& \beta^{1}=\left(\sin \left(y_{3}\right) d x_{1}-\cos \left(y_{3}\right) d x_{2}\right) / \sqrt{3}, \\
& \beta^{2}=\left(\cos \left(y_{3}\right) d x_{1}+\sin \left(y_{3}\right) d x_{2}\right) / \sqrt{3}, \\
& \beta^{3}=\left(\cos \left(2 y_{3}\right) d x_{4}-\sin \left(2 y_{3}\right) d x_{3}\right) /(2 \theta) .
\end{aligned}
$$

We now take the expansion (4.1), which gives $\delta A_{\kappa}$, and apply the transition function (2.15). This transforms the Lie algebra of $H=S O(3)$ as follows:

$$
\begin{aligned}
& l_{1} \mapsto \cos \left(r y_{3}\right) l_{1}-\sin \left(r y_{3}\right) l_{2} \equiv \tilde{l}_{1}, \\
& l_{2} \mapsto \sin \left(r y_{3}\right) l_{1}+\cos \left(r y_{3}\right) l_{2} \equiv \tilde{l}_{2}, \\
& l_{3} \mapsto l_{3} \equiv \tilde{l}_{3} .
\end{aligned}
$$

As a result,

$$
\delta A_{\delta}=\sum_{i, j} \alpha_{i j}(\theta, g) \beta^{i} \otimes \tilde{l}_{j}+\sum_{i} \gamma_{i}(\theta, g) d \theta \otimes \tilde{l}_{i}
$$

Making the substitutions (4.12) for the 1-forms and (4.13) for the Lie algebra, we have an expansion of $\delta A_{\delta}$ in terms of $d x_{i} \otimes l_{j}$. Comparing to (4.9) gives $\delta A_{i}^{j}$ in terms of $\alpha$ and $\gamma$. Inverting this relationship we get that

$$
\begin{aligned}
\alpha_{23}= & \sqrt{3}\left[\delta A_{1}^{3} \cos \left(y_{3}\right)+\delta A_{2}^{3} \sin \left(y_{3}\right)\right]+O(\theta), \\
\alpha_{13}= & \sqrt{3}\left[\delta A_{1}^{3} \sin \left(y_{3}\right)-\delta A_{2}^{3} \cos \left(y_{3}\right)\right]+O(\theta), \\
\alpha_{33} /(2 \theta)= & \delta A_{4}^{3} \cos \left(2 y_{3}\right)-\delta A_{3}^{3} \sin \left(2 y_{3}\right)+O(\theta), \\
\gamma_{3}= & \delta A_{3}^{3} \cos \left(2 y_{3}\right)+\delta A_{4}^{3} \sin \left(2 y_{3}\right)+O(\theta), \\
\alpha_{22}-\alpha_{11}= & \sqrt{3}\left[\left(\delta A_{1}^{2}+\delta A_{2}^{1}\right) \cos \left((r-1) y_{3}\right)\right. \\
& \left.+\left(\delta A_{1}^{1}-\delta A_{2}^{2}\right) \sin \left((r-1) y_{3}\right)\right]+O(\theta), \\
\alpha_{12}+\alpha_{21}= & \sqrt{3}\left[\left(\delta A_{1}^{1}-\delta A_{2}^{2}\right) \cos \left((r-1) y_{3}\right)\right. \\
& \left.-\left(\delta A_{1}^{2}+\delta A_{2}^{1}\right) \sin \left((r-1) y_{3}\right)\right]+O(\theta), \\
\alpha_{22}+\alpha_{11}= & \sqrt{3}\left[\left(\delta A_{1}^{2}-\delta A_{2}^{1}\right) \cos \left((r+1) y_{3}\right)\right. \\
& \left.+\left(\delta A_{1}^{1}+\delta A_{2}^{2}\right) \sin \left((r+1) y_{3}\right)\right]+O(\theta), \\
\alpha_{12}-\alpha_{21}= & \sqrt{3}\left[-\left(\delta A_{1}^{1}+\delta A_{2}^{2}\right) \cos \left((r+1) y_{3}\right)\right. \\
& \left.+\left(\delta A_{1}^{2}-\delta A_{2}^{1}\right) \sin \left((r+1) y_{3}\right)\right]+O(\theta),
\end{aligned}
$$




$$
\begin{aligned}
\alpha_{31} /(2 \theta)+\gamma_{2}= & \left(\delta A_{4}^{1}+\delta A_{3}^{2}\right) \cos \left((r-2) y_{3}\right) \\
& -\left(\delta A_{4}^{2}-\delta A_{3}^{1}\right) \sin \left((r-2) y_{3}\right)+O(\theta), \\
\alpha_{32} /(2 \theta)-\gamma_{1}= & \left(\delta A_{4}^{2}-\delta A_{3}^{1}\right) \cos \left((r-2) y_{3}\right) \\
& +\left(\delta A_{4}^{1}+\delta A_{3}^{2}\right) \sin \left((r-2) y_{3}\right)+O(\theta), \\
\alpha_{31} /(2 \theta)-\gamma_{2}= & \left(\delta A_{4}^{1}-\delta A_{3}^{2}\right) \cos \left((r+2) y_{3}\right) \\
& -\left(\delta A_{4}^{2}+\delta A_{3}^{1}\right) \sin \left((r+2) y_{3}\right)+O(\theta), \\
\alpha_{32} /(2 \theta)+\gamma_{1}= & \left(\delta A_{4}^{2}+\delta A_{3}^{1}\right) \cos \left((r+2) y_{3}\right) \\
& +\left(\delta A_{4}^{1}-\delta A_{3}^{2}\right) \sin \left((r+2) y_{3}\right)+O(\theta) .
\end{aligned}
$$

The first two equations give rise to mode 1 , the next two to mode 2 , and so on.

We next wish to study what boundary conditions to apply to deformations of $(r, t)$ YM connections for which $r$ is not an odd integer. Modes 3-6 are clearly impossible, so the natural choice of boundary conditions is to require that, with the exception of the modes 1 and 2, all components of $\alpha$ and $\gamma$ be zero at $\theta=0$, and $\alpha_{3 j}^{m \prime}(0)=0$ for all $j, m$. We call a deformation that meets these conditions regular at 0 . If in addition all components $\alpha, \gamma$ and their linear combinations grow or decay as a power of $\theta$ near $\theta=0$, then we say the deformation is power-law regular.

Power-law regularity is very similar to a condition that Johan Råde recently proved ([R1], Theorem 2). Råde showed that, locally, a finite action YM connection with a non-removable holonomy singularity is gauge-equivalent to a connection for which the "direct" components of the connection (in our notation $\left(r-a_{3}\right) / f_{3}, \alpha_{13}, \alpha_{23}, \gamma_{3}$, and $\left.\alpha_{33} / f_{3}\right)$ are at most $O\left(\theta^{0}\right)$, while the remaining components are a positive power of $\theta$ smaller. The Rade estimates are slightly weaker than power-law regularity, since they allow all components of $\alpha_{i 3}$ and $\gamma_{3}$ to be $O\left(\theta^{0 \text { or } 1}\right)$, not just the particular components of exceptional modes 1 and 2 . The other difference is that Råde's gauge condition is slightly different from $d_{A}^{*}(\delta A)=0$.

A weaker, but still reasonable, set of boundary conditions is to allow components of $\gamma$ to have integrable singularities at $\theta=0$, while requiring all components of $\alpha$ (excepting mode 1 ) to go to zero at $\theta=0$. We call a deformation that satisfies these conditions weakly regular, and if it also exhibits power-law growth we call it weakly power-law regular.

Proposition 4.6. If $A$ is a finite-action $(r, t) \mathrm{YM}$ connection and if $\delta A$ is power-law regular, then for all real $\tau, A+\tau \delta A$ has square-integrable curvature near $\theta=0$.

Proof. Curvature is quadratic in the connection, so $F_{A+\tau \delta A}=F_{A}+\tau \delta F+\tau^{2} \delta^{2} F$. Since $A$ has finite action, $F_{A}$ is in $L^{2}$. So $F_{A+\tau \delta A}$ is in $L^{2}$ for all $\tau$ if $\delta F$ and $\delta^{2} F$ are.

If $\delta A$ is power-law regular, then there exists a constant $s>0$ such that all components of $\alpha_{1 j}, \alpha_{2 j}, \gamma_{j}$ and $\alpha_{3 j} / \theta$ are $O\left(\theta^{s}\right)$, except for those of modes 1 and 2 , which are constant $+O\left(\theta^{s}\right)$. This makes all the (03i), (23i), and (31i) components of $\delta F$ be $O\left(\theta^{s}\right)$ (the $O(1)$ components from modes 1 and 2 cancel). Since $1 / G_{1}, 1 / G_{2}$, and $G_{3}$ are $O\left(\theta^{-1}\right)$, the expressions

$$
\left|F_{23 i}\right|^{2} / G_{1}, \quad\left|F_{31 i}\right|^{2} / G_{2}, \quad\left|F_{03 i}\right|^{2} G_{3}
$$


are $O\left(\theta^{2 s-1}\right)$, and so are integrable near zero. Similarly, all the (01i), (02i), and (12i) components of $\delta F$ are $O\left(\theta^{s-1}\right)$. When squared and multiplied by an $O(\theta)$ metric factor, this again gives $O\left(\theta^{2 s-1}\right)$, which is integrable. Thus $\delta F$ is square-integrable near $\theta=0$.

$\delta^{2} F$ is even easier. Since both special modes involve $l_{3}$, each term in $\delta^{2} F$ has at least one factor that is not from a special mode, and so is $O\left(\theta^{s}\right)$. Thus $\delta^{2} F$ is squareintegrable.

Unfortunately, the converse of Proposition 4.6 is false. One can find finiteenergy deformations $\delta A$ that are arbitrarily singular, simply by applying an arbitrarily singular infinitesimal gauge transformation to $A$. This problem did not arise in the smooth case (Proposition 4.5), as a gauge transformation there would have to take on a definite (and finite) value at $\theta=0$. However, if $r$ is not an odd integer we work on the open manifold $X$ that does not contain $Q_{0}$, so we have no a priori control on gauge transformations near $\theta=0$.

Even applying a gauge-fixing condition such as $d_{A}^{*}(\delta A)=0$ does not fix the problem, since the equation $d_{A}^{*} d_{A} \phi=0$, where $\phi$ is an infinitesimal gauge transformation on $P_{X}$, is not elliptic. To control the problem we have to limit the behavior of $\phi$ near $\theta=0$ by hand. Since for gauge transformations $\gamma=\phi^{\prime}$, this translates into controlling $\gamma$. Requiring $\phi$ to have a definite limit as $\theta \rightarrow 0$ corresponds to requiring $\gamma$ to be integrable, which leads to weak regularity. If we further require $\gamma$ to be $O(1)$ we get the stronger notion of regularity.

Proposition 4.7. Suppose $r \geqq 0$ is not an odd integer. Let $A$ be a finite-action equivariant $(r, t)$ connection that is a solution of the Yang-Mills equations, and let $\delta A$ be a deformation of $A$ such that $d_{A}^{*}(\delta A)=0$, such that $\delta F$ is square-integrable on $X$, and such that $\delta A$ has power-law growth near $\theta=0$. Then, if the $\gamma$ components of $\delta A$ are integrable near $\theta=0, \delta A$ is weakly power-law regular near $\theta=0$. If the $\gamma$ components of $\delta A$ are $O(1), \delta A$ is power-law regular near $\theta=0$.

Proof. We sequentially prove the following six statements. The first two are mild regularity results, which we then use to prove four stronger results, which imply the theorem.

1. All components of all the $\alpha_{i j}$ 's are $O(1)$.

2. All components of all the $\alpha_{3 i}$ 's are $O\left(\theta^{s}\right)$ for some positive constant $s$.

3. All components of $\alpha_{11}, \alpha_{22}, \alpha_{12}$ and $\alpha_{21}$ are $o(1)$.

4. If $\gamma$ is $O(1)$ then all components of $\alpha_{31}, \alpha_{32}, \theta \gamma_{1}$ and $\theta \gamma_{2}$ are $o(\theta)$.

5. All components of $\alpha_{33}$ and $\theta \gamma_{3}$ are $o(\theta)$, with the exception of mode 2, which may be $O(\theta)$.

6. All components of $\alpha_{13}$ and $\alpha_{23}$ are $o(1)$, with the possible exception of mode 1 , which may be $O(1)$.

Note that, if $\gamma$ is integrable and exhibits power-law behavior, it must go as $\theta^{s-1}$ for some positive $s$.

Since $G_{3}, 1 / G_{1}$ and $1 / G_{2}$ all have simple poles at $\theta=0$, the square-integrability of $\delta F$ implies that all the (03i), (23i), and (31i) components of $\delta F$ are $o(1)$, and that the remaining components are $o\left(\theta^{-1}\right)$. Furthermore, the three components of $d_{A}^{*}(\delta A)$ are identically zero. We will control the various components of $\alpha$ and $\gamma$ by the corresponding components of $\delta F$ and $d_{A}^{*}(\delta A)$.

Since all the (0ij) components of $\delta F$ are at worst $o\left(\theta^{-1}\right)$, and since $\gamma$ is $o\left(\theta^{-1}\right)$, all components of $\alpha_{i j}^{\prime}$ are at worst $o\left(\theta^{-1}\right)$, which implies statement 1 . 
Next we look at the components of $d_{A}^{*}(\delta A)$. All the terms involving $\alpha_{1 i}$ and $\alpha_{2 i}$ are $O(1)$, and the terms involving $\gamma_{i}$ are $O\left(\theta^{s-2}\right)$, so the remaining terms must also be $O\left(\theta^{s-2}\right)$. This implies that for any odd $m,-m \alpha_{31}^{m}+r \alpha_{32}^{m}$ and $m \alpha_{32}^{m}-r \alpha_{31}$ are $O\left(\theta^{s}\right)$, and for any even $m, m \alpha_{33}^{m}$ is $O\left(\theta^{s}\right)$. Since $r$ is not an odd integer, this proves that all components of $\alpha_{3 i}$ are $O\left(\theta^{s}\right)$, with the possible exception of $\alpha_{33}^{0}$.

To control $\alpha_{33}^{0}$ we look at the (033) component of $\delta F$, namely $\alpha_{33}^{\prime}-l_{3} \gamma_{3}$. Since this is $o(1)$, and since $l_{3} \Psi_{l, 0}=0, \alpha_{33}^{0^{\prime}}$ must be $o(1)$, so $\alpha_{33}^{0}$ is a constant plus $o(\theta)$. However, by the Sibners' theorem, the holonomy around $S_{+}$must be constant, so $\alpha_{33}^{0}(0) \Psi_{l 0}$ cannot depend on $y_{1}$ or $y_{2}$. If $l>0$, this implies that the constant part of $\alpha_{33}^{0}$ vanishes. If $l=0$, a deformation $\alpha_{33}(0) \neq 0$ can have finite energy, but it is a change in the value of $r$. For fixed $r$, this is inadmissible.

We next prove statement 3 . We look at the $\Psi_{l, m}$ components of the (231), (232), (311) and (312) components of $\delta F$. These components are equal to

$$
-m \alpha_{21}^{m}-\alpha_{11}^{m}-r \alpha_{22}^{m}, \quad m \alpha_{22}^{m}-\alpha_{12}^{m}+r \alpha_{21}^{m}, \quad-m \alpha_{11}^{m}-\alpha_{21}^{m}+r \alpha_{12}^{m}, \quad m \alpha_{12}^{m}-\alpha_{22}^{m}-r \alpha_{11}^{m} \text {, }
$$

plus terms that, by statements 1 and 2, are $o(1)$. Since $m$ is even and $r$ is not odd, these four expressions are linearly independent, so the only way for these four components of $\delta F$ to be $o(1)$ is for $\alpha_{11,12,21,22}^{m}$ to all be $o(1)$.

Statement 4 is similar. We look at the $l_{1}$ and $l_{2}$ components of $d_{A}^{*}(\delta A)$ and the (031) and (032) components of $\delta F$. To order $O(\theta)$ we have that

$$
\alpha_{31}^{m}-m \theta \gamma_{1}^{m}-r \theta \gamma_{2}^{m}, \quad \alpha_{32}^{m}+m \theta \gamma_{2}^{m}+r \theta \gamma_{1}^{m}, \quad 4 \theta \gamma_{1}^{m}-m \alpha_{31}^{m}+r \alpha_{32}^{m}, \quad 4 \theta \gamma_{2}^{m}+m \alpha_{32}^{m}-r \alpha_{31}^{m}
$$

are all zero. Since $r$ is not an odd integer, these four expressions are linearly independent, and so all components of $\theta \gamma_{1}, \theta \gamma_{1}, \alpha_{31}^{m}$, and $\alpha_{32}^{m}$ must be $o(\theta)$.

To prove statement 5 we look at the $l_{3}$ component of $d_{A}^{*}(\delta A)$ and the $(033)$ component of $\delta F$. To order $O(\theta)$ we have

$$
\alpha_{33}^{m}-m \theta \gamma_{3}^{m}=4 \theta \gamma_{3}^{m}-m \alpha_{33}^{m}=0 \text {. }
$$

If $m \neq 2$ these are linearly independent, implying that $\alpha_{33}^{m}$ and $\theta \gamma_{3}^{m}$ are $o(\theta)$. If $m=2$ the two equations are linearly dependent, and we find $2 \gamma_{3}^{2}(0)=\alpha_{33}^{2^{\prime}}(0)$ may be nonzero. This is mode 2 .

To prove statement 6 we look at the (233) and (313) components of $\delta F$ to order $O(1)$. For $m \neq 1$ the expressions are linearly independent, so all components are $o(1)$. For $m=1$ the two expressions are linearly dependent, and we get mode 1 .

4.3 Solutions to the Anti-Self-Duality Equations. In this section we look for solutions to the linearized (anti)self-duality equations (4.3), restricted to a particular representation of $S O(3)$, near $\theta=0$ and $\theta=\pi / 3$. We make repeated use of the following standard fact about solutions to an ODE system near a regular singular point.

Proposition 4.8. Let $x$ be a real variable, $y(x)$ an $n$-vector, and $M$ a fixed $n \times n$ matrix. Then the n-dimensional space of solutions to the ODE

$$
x d y / d x=M y
$$

on $(0, \varepsilon)$ is spanned by functions of the form

$$
y(x)=\xi x^{\lambda},
$$


where $\xi$ is an eigenvector of $M$ with eigenvalue $\lambda$. If we add a forcing term to the right-hand side,

$$
x d y / d x=M y+\xi x^{s},
$$

then a particular solution is $y=\xi x^{s} /(s-\lambda)$ if $s \neq \lambda$ or $y=\xi x^{s} \log (x)$ if $s=\lambda$.

The number of solutions to (4.20) that are regular near $x=0$ depends only on the eigenvalues of $M$. Each positive eigenvalue gives a solution that vanishes at $x=0$, each zero eigenvalue gives a solution that is nonzero but finite at $x=0$, and each negative eigenvalue gives a solution that diverges at $x=0$. If $s>0$ in (4.22), then the particular solution vanishes at $x=0$.

We can find the dimension of the space of solutions to the anti-self-duality equations near $\theta=0$ by studying the leading-order terms. The leading-order parts of the ASD equations (4.3) and the gauge-fixing equations are

$$
\begin{aligned}
& \theta \alpha_{11}^{\prime}=\left(-l_{3} \alpha_{21}-\alpha_{11}-r \alpha_{22}\right) / 2, \\
& \theta \alpha_{12}^{\prime}=\left(-l_{3} \alpha_{22}-\alpha_{12}+r \alpha_{21}\right) / 2, \\
& \theta \alpha_{13}^{\prime}=\left(-l_{3} \alpha_{23}-\alpha_{13}\right) / 2, \\
& \theta \alpha_{21}^{\prime}=\left(l_{3} \alpha_{11}-\alpha_{21}+r \alpha_{12}\right) / 2, \\
& \theta \alpha_{22}^{\prime}=\left(l_{3} \alpha_{12}-\alpha_{22}-r \alpha_{11}\right) / 2, \\
& \theta \alpha_{23}^{\prime}=\left(l_{3} \alpha_{13}-\alpha_{23}\right) / 2, \\
& \theta \alpha_{31}^{\prime}=\left(l_{3} \Gamma_{1}+r \Gamma_{2}\right) / 2, \\
& \theta \alpha_{32}^{\prime}=\left(l_{3} \Gamma_{2}-r \Gamma_{1}\right) / 2, \\
& \theta \alpha_{33}^{\prime}=l_{3} \Gamma_{3} / 2, \\
& \theta \Gamma_{1}^{\prime}=\left(-l_{3} \alpha_{31}-r \alpha_{32}\right) / 2, \\
& \theta \Gamma_{2}^{\prime}=\left(-l_{3} \alpha_{32}+r \alpha_{31}\right) / 2, \\
& \theta \Gamma_{3}^{\prime}=-l_{3} \alpha_{33} / 2,
\end{aligned}
$$

where $\Gamma_{i} \equiv f_{3} \gamma_{i}$.

The exact equations differ from (4.23) as follows. There are $O(1) \alpha_{3 i}, O(1) \Gamma_{i}$, $O(\theta) \alpha_{1 i}$ and $O(\theta) \alpha_{2 i}$ corrections to the expressions for $\theta \alpha_{1 i}^{\prime}$ and $\theta \alpha_{2 i}^{\prime}$. There are $O\left(\theta^{2}\right) \alpha_{3 i}, O\left(\theta^{2}\right) \Gamma_{i}, O\left(\theta^{2}\right) \alpha_{1 i}$ and $O\left(\theta^{2}\right) \alpha_{2 i}$ corrections to the expressions for $\theta \alpha_{3 i}^{\prime}$ and $\theta \Gamma_{i}^{\prime}$. Some of these errors come from our having replaced

$$
\begin{aligned}
a_{3} & =r+O\left(\theta^{2}\right), \\
f_{3} & =2 \theta+O\left(\theta^{3}\right), \\
f_{1} f_{2} & =3+O\left(\theta^{2}\right), \\
f_{1} / f_{2} & =1+O(\theta),
\end{aligned}
$$

with their leading order terms. Others come from our having neglected the $\alpha_{3 i}$ and $\Gamma_{i}$ contributions to the derivatives of $\alpha_{1 i}$ or $\alpha_{2 i}$, and vice-versa. We shall see that these higher-order corrections make no essential difference. 
The ODE system (4.23) decomposes into 4 uncoupled subsystems, one for $\alpha_{33}$ and $\Gamma_{3}$, one for $\alpha_{31}, \alpha_{32}, \Gamma_{1}$ and $\Gamma_{2}$, one for $\alpha_{13}$ and $\alpha_{23}$, and one for $\alpha_{11}, \alpha_{21}$, $\alpha_{12}$ and $\alpha_{22}$. Moreover, $l_{1}$ and $l_{2}$ do not appear, so we can solve the equations separately for each value of $m$. Using Proposition 4.8 and Eq. (2.30), we get

Proposition 4.9. The solutions to Eqs. (4.23) are spanned by the following:

1. $\alpha_{33}^{m}=\Gamma_{3}^{m}=\theta^{m / 2}, m>0$ even,

2. $\alpha_{33}^{m}=-\Gamma_{3}^{m}=\theta^{-m / 2}, m>0$ even,

3. $\alpha_{31}^{m}=\alpha_{32}^{m}=\Gamma_{1}^{m}=-\Gamma_{2}^{m}=\theta^{(m-r) / 2}, \quad m>0$ odd ,

4. $\alpha_{31}^{m}=\alpha_{32}^{m}=-\Gamma_{1}^{m}=\Gamma_{2}^{m}=\theta^{(r-m) / 2}, \quad m>0$ odd ,

5. $\alpha_{31}^{m}=-\alpha_{32}^{m}=\Gamma_{1}^{m}=\Gamma_{2}^{m}=\theta^{(r+m) / 2}, \quad m>0$ odd ,

6. $\alpha_{31}^{m}=-\alpha_{32}^{m}=-\Gamma_{1}^{m}=-\Gamma_{2}^{m}=\theta^{-(r+m) / 2}, \quad m>0$ odd ,

7. $\alpha_{13}^{m}=\alpha_{23}^{m}=\theta^{-(m+1) / 2}, \quad m>0$ odd ,

8. $\alpha_{13}^{m}=-\alpha_{23}^{m}=\theta^{(m-1) / 2}, \quad m>0$ odd ,

9. $\alpha_{11}^{m}=\alpha_{12}^{m}=-\alpha_{21}^{m}=\alpha_{22}^{m}=\theta^{(m-r-1) / 2}, \quad m>0$ even,

10. $\alpha_{11}^{m}=-\alpha_{12}^{m}=\alpha_{21}^{m}=\alpha_{22}^{m}=\theta^{-(m+r+1) / 2}, \quad m>0$ even,

11. $\alpha_{11}^{m}=\alpha_{12}^{m}=\alpha_{21}^{m}=-\alpha_{22}^{m}=\theta^{(r-m-1) / 2}, \quad m>0$ even,

12. $-\alpha_{11}^{m}=\alpha_{12}^{m}=\alpha_{21}^{m}=\alpha_{22}^{m}=\theta^{(r+m-1) / 2}, \quad m>0$ even .

If $l$ is even there are the solutions

13. $\alpha_{33}^{0}=1$,

14. $\alpha_{11}^{0}=\alpha_{22}^{0}=\theta^{-(r+1) / 2}$,

15. $\alpha_{11}^{0}=-\alpha_{22}^{0}=\theta^{(r-1) / 2}$,

while if $l$ is odd we have

$13^{\prime} . \Gamma_{3}^{0}=1$,

14. $\alpha_{12}^{0}=\alpha_{21}^{0}=\theta^{(r-1) / 2}$,

15'. $\alpha_{12}^{0}=-\alpha_{21}^{0}=\theta^{-(r+1) / 2}$.

We are also interested in solutions to the ASD equations near $\theta=\pi / 3$ for $t \leqq 1$. These are equivalent to solutions of the self-duality equations near $\theta=0$ for $r \leqq 1$. To leading order, the self-duality and gauge-fixing equations for $\alpha_{3 i}^{\prime}$ and $\Gamma_{i}^{\prime}$ are the same as the ASD and gauge-fixing equations. The leading order SD expressions for $\alpha_{1 i}^{\prime}$ and $\alpha_{2 i}^{\prime}$ are minus those of (4.23). The solutions to the leading-order SD equations are therefore similar to those given by Proposition 4.9, the difference being that the exponents in solutions 7-15 (or 7-15') flip sign.

Proposition 4.10. The regular (or weakly regular) solutions to the linearized antiself-duality equations (4.3) with the gauge condition $d_{A}^{*}(\delta A)=0$ near $\theta=0$ are in 1-1 correspondence with regular (or weakly regular) solutions to (4.23). 
Proof. We must show that the higher-order terms neglected in Eq. (4.23) do not affect regularity. By the general theory of ODEs, the behavior of any linear ODE system near a regular singular point is controlled by the leading-order terms in the equation. One solves the leading-order equation, then uses the solution to compute the correction terms, then solves the equation again using the correction terms as a source, and continues iterating. The iterative procedure converges, giving a solution to the full system that has the same growth behavior near the singular point as the first solution to the leading-order equation.

The only potential trouble in our case comes from the $O(1) \alpha_{33}$ and $O(1) \Gamma_{i}$ corrections to $\theta \alpha_{1 i}^{\prime}$ and $\theta \alpha_{2 i}^{\prime}$ and the $O\left(\theta^{2}\right) \alpha_{1 i}$ and $O\left(\theta^{2}\right) \alpha_{2 i}$ corrections to $\theta \alpha_{33}^{\prime}$ and $\theta \Gamma_{i}^{\prime}$. It is not immediately obvious that these terms are really lower order.

However, if a solution to (4.23) is regular, then $\alpha_{33}$ and $\Gamma_{i}$ are at most $O(\theta)$. Treating these as source terms for the equations for $\alpha_{1 i}^{\prime}$ and $\alpha_{2 i}^{\prime}$, we get (by Proposition 4.8) that $\alpha_{1 i}$ and $\alpha_{2 i}$ change by at most $O(\theta \log (\theta))$, which does not affect their regularity. Similarly, if a solution to (4.23) is weakly regular, then $\alpha_{33}$ and $\Gamma_{i}$ are at most $O\left(\theta^{s}\right)$ for some $s>0$, so $\alpha_{1 i}$ and $\alpha_{2 i}$ change by at most $O\left(\theta^{s} \log (\theta)\right)$. Also if a solution is regular or weakly regular, then $a_{1 i}$ and $a_{2 i}$ are at most $O(1)$, so their contribution to $\alpha_{33}$ and $\Gamma_{i}$ is at most $O\left(\theta^{2} \log (\theta)\right)$, which again does not affect regularity or weak regularity.

4.4 Dimensions of Solution Spaces. For a given representation of $S O(3)$, there are $6 l+3$ linearly independent solutions to the ASD and gauge-fixing equations, since these constitute a $1^{\text {st }}$ order system of ODEs in the $6 l+3$ variables $\alpha_{i j}^{m}$ and $\gamma_{i}^{m}$. We will compute the dimension $N_{+}\left(N_{+}^{w}\right)$ of the space of solutions that are regular (weakly regular) at $\theta=0$, and the dimension $N_{-}\left(N_{-}^{w}\right)$ of the space of solutions that satisfy the boundary conditions at $\theta=\pi / 3$. When $r$ is an odd integer we apply the smooth boundary conditions at $\theta=0$, so in those cases $N_{+}=N_{+}^{w}$. Generically, the dimension $N(l)$ of the space of solutions that meet the boundary conditions at both endpoints will be $N_{+}+N_{-}-6 l-3$ (or 0 dimensional if $N_{+}+N_{-}<6 l+3$ ). We can then sum this number over representations of $S O(3)$ to get the dimension of the space of allowable infinitesimal deformations. Generically, this equals the dimension of the moduli space. ${ }^{2}$

We begin by computing $N_{+}$and $N_{+}^{w}$ (Proposition 4.11) and then compute $N_{-}$and $N^{w}$ (Proposition 4.12). Let $\{x\}$ denote the greatest odd integer less than or equal to $x$.

Proposition 4.11. Suppose $l \geqq 1$, and suppose $r$ is not an odd integer. Then the number of regular solutions is $N_{+}=3 l+1$ for $r>\{l+2\}$, and $N_{+}<3 l+1$ for $1<r<\{l+2\}$. The number of weakly regular solutions is $N_{+}^{w}=3 l+1$ for $r>\{l+1\}$, and $N_{+}^{w}=3 l$ for $1<r<\{l+1\}$.

If $l \geqq 1$ and $r$ is an odd integer, then the number of solutions with the boundary conditions of Prop. 4.5 is $N_{+}=N_{+}^{w}=3 l+1$ for $r>l$, and $N_{+}=N_{+}^{w}=3 l$ for $r \leqq l$.

Proof. We first consider $N_{+}$for $l \geqq 2$ even, and evaluate solutions according to the classification of Proposition 4.9. There are of course $l / 2$ positive even values of $m$, the largest being $l$, and there are $l / 2$ positive odd values of $m$, the largest being $l-1$. So there are $l / 2$ solutions of each type $1-12$, and 1 solution of each type 13-15.

${ }^{2}$ We cannot be certain that the round metric on $S^{4}$ gives generic behavior. We may have to vary our metric functions $f_{1}, f_{2}$, and $f_{3}$ in a generic way away from the endpoints. 
For $r>l+1$, all the type $1,4,5,8,11$, and 12 solutions are regular at $\theta=0$, as all have sufficiently large powers of $\theta$ for all values of $m$. None of the type 2, 3, 6, 7, 9, and 10 solutions are regular, as they all carry negative powers of $\theta$. In addition, solution 15 is regular, while 13 and 14 are not, giving a total of $3 l+1$ regular solutions.

When $r=l+1$ the counting is the same. The $m=l-1$ case of type 4 goes as $\theta^{1}$, but this is allowed by exceptional mode 5 of Proposition 4.5. Similarly, the $m=l$ case of type 11 goes as $\theta^{0}$, but this is exceptional mode 3 . However, when $r$ drops below $l+1$, these two modes cease being regular, and $N_{+}$drops down to $3 l-1$.

Decreasing $r$ further, $N_{+}$remains $3 l-1$ until $r$ hits $l-1$. At that point the $m=l$ case of type 9 becomes regular (exceptional mode 4), and $N_{+}=3 l$. However, once $r$ drops below $l-1$ the $m=l-3$ case of type 4 and the $m=l-2$ case of type 11 cease being regular, and $N_{+}$drops to $3 l-2$.

As $r$ decreases further, the pattern repeats itself. Whenever $r$ hits an odd integer $\leqq l-3$, two modes become regular. One is of type 3 and corresponds to exceptional mode 6 , while the other is of type 9 and corresponds to exceptional mode $4 . N_{+}$thus increases to $3 l$. However, once $r$ drops below the odd integer two other modes, one of type 4 and one of type 11 , cease being regular, reducing $N_{+}$back down to $3 l-2$. In any case, $N_{+}$is only bigger than $3 l$ when $r \geqq l+1=\{l+2\}$.

We next consider $N_{+}$for $l \geqq 3$ odd. There are $(l+1) / 2$ positive odd values of $m$ and $(l-1) / 2$ positive even values of $m$.

For $r \geqq l+2$, all the type $1,4,5,8,11$, and 12 solutions are regular at $\theta=0$, while none of the type $2,3,6,7,9$, and 10 solutions are regular. In addition, solution $14^{\prime}$ is regular while $13^{\prime}$ and $15^{\prime}$ are not. This makes for a total of $N_{+}=3 l+1$.

When $r$ drops below $l+2$, the $m=l$ case of type 4 ceases to be regular, reducing $N_{+}$to $3 l$, where it remains through $r=l$. When $r$ drops below $l$, the $m=l-2$ case of type 4 and the $m=l-1$ case of type 11 cease being regular, reducing $N_{+}$to $3 l-2$. When $r$ hits $l-2$, and at every odd integer thereafter, a type 3 solution and a type 9 solution become regular, increasing $N_{+}$to $3 l$. However, once $r$ drops below the odd integer, a type 4 solution and a type 11 solution cease being regular, reducing $N_{+}$to $3 l-2$ again. Again, $N_{+}$is only bigger than $3 l$ when $r \geqq l+2=\{l+2\}$.

We next consider $N_{+}$for $l=1$. For $l=1$, solutions of type 1,2 , or $9-12$ do not exist. The type 5,8 , and $14^{\prime}$ solutions are always regular, the type 4 solution is regular for $r \geqq 3$, and the type $3,6,7,13^{\prime}$, and $15^{\prime}$ solutions are never regular. So $N_{+}=4=3 l+1$ for $r \geqq 3$ and $N_{+}=3<3 l+1$ for $r<3$.

For $N_{+}^{w}$ the counting is the same, with the following exceptions. For $l$ even, when $l-1<r<l+1$ the $m=l-1$ type 4 solution is weakly regular but not regular, which increases $N_{+}^{w}$ from $3 l-1$ to $3 l$. When $r<l-1$ there are 2 solutions, one of type 3 and one of type 4, that are weakly regular but not regular. This increases $N_{+}^{w}$ from $3 l-2$ to $3 l$. Thus, for even $l, N_{+}^{w}=3 l+1$ when $r>l+1$ and $N_{+}^{w}=3 l$ when $r<l+1$.

When $l$ is odd and $l<r<l+2$, the $m=l$ type 4 solution is weakly regular but not regular, so $N_{+}^{w}=3 l+1$ rather than $3 l$. When $r<l$ there are two weakly regular but not regular solutions, one of type 3 and one of type 4 , so $N_{+}^{w}=3 l$ rather than $3 l-2$.

Proposition 4.12. If $t<1$ and $l \geqq 1$, then $N_{-}<N_{-}^{w}=3 l+3$. If $t=1$ and $l \geqq 2$, then $N_{-}=N_{-}^{w}=3 l+3$. If $t=l=1$, then $N_{-}=N_{-}^{w}<3 l+3$. 
Proof. We proceed as in the comment after Proposition 4.9. Anti-self-dual solutions near $\theta=\pi / 3$ are equivalent to self-dual solutions near $\theta=0$, with the roles of $r$ and $t$ interchanged. These are given by Proposition 4.9, only with the exponents on modes $7-15$ reversed.

If $t=1$ and $l>1$, then the regular solutions are all the type 1 solutions, all but $m=1$ of the type 3 solutions, all the type 5 solutions, all the type 7 solutions, the $m=1$ type 8 solution, then $m=2$ type 9 solution, all the type 10 and type 11 solutions, and the type 14 and 15 (or $14^{\prime}$ and $15^{\prime}$ ) solutions. This adds up to $3 l+3$ regular solutions.

If $t<1$ and $l>1$, then the $m=2$ type 9 solution and the $m=1$ type 5 solution are no longer regular, and $N_{-}$drops to $3 l+1$. The type 3 and type 5 solutions with $m=1$ are weakly regular but not regular, so $N_{-}^{w}=N_{-}+2=3 l+3$.

If $l=1$ and $t=1$ then the type $5,7,8,14^{\prime}$ and $15^{\prime}$ solutions are regular, so $N_{-}=N_{-}^{w}=5$. If $l=1$ and $t<1$ then the type 5 solution is no longer regular and $N_{-}=4$. Once again, the type 3 and type 5 solutions with $m=1$ are weakly regular but not regular, so $N_{-}^{w}=N_{-}+2=6$.

Theorem 4.13. For generic metrics and regular boundary conditions, $N(l)=1$ if $l \geqq 2$, $r \geqq\{l+2\}$, and $t=1$, and $N(l)=0$ otherwise.

Proof. If $l \geqq 2, r \geqq\{l+2\}$, and $t=1$, then $N_{+}=3 l+1$ and $N_{-}=3 l+3$, so for generic metrics $N(\bar{l})=N_{+}+N_{-}-(6 l+3)=1$. If $r<\{l+2\}$ then $N_{+}<3 l+1$, so $N(l)<1$. If $l=1$ or $t<1$ then $N_{-}<3 l+3$, so again $N(l)<1$. Finally, if $l=0, N_{+}=1$ (type 15 ) and $N_{-}$is at most 2 (types 14 and 15 ), so $N(0)=0$.

A similar addition gives

Theorem 4.14. For generic metrics and weakly regular boundary conditions, and for $r$ not an odd integer, $N^{w}(l)=1$ if $l>1$ and $r>\{l+1\}$, and $N^{w}(l)=0$ otherwise. For $l=1, N^{w}(1)=1$ if $t<1$ and $N^{w}(1)=0$ if $t=1$.

Proof of Theorem 1.2. By Theorem 4.13, $N(l)=1$ for all $l$ between 2 and $\{r\}-1$, and equals 0 for all other values of $l$. By the discussion before Proposition 2.1, the spin-l representation appears $2 l+1$ times in the decomposition of $L^{2}(S O(3))$, and the anti-self-duality equations are the same for each appearance. Thus the total number of regular solutions to the linearized anti-self-duality equations is

$$
\sum_{l=0}^{\infty}(2 l+1) N(l)=\sum_{l=2}^{\{r\}-1}(2 l+1)=\{r\}^{2}-4 .
$$

For a generic metric this equals the dimension of the moduli space.

Proof of Theorem 1.3. If $t=1$, the total number of weakly regular solutions to the linearized anti-self-duality equations is

$$
\sum_{l=0}^{\infty}(2 l+1) N^{w}(l)=\sum_{l=2}^{\{r\}}(2 l+1)=(\{r\}+1)^{2}-4 .
$$

If $t<1$ we must add on the contribution of the $l=1$ representation to get $(\{r\}+1)^{2}-1$. 


\section{Non-self-dual Connections}

In this section we consider equivariant non-self-dual connections without holonomy. That is, we consider the case where $r$ and $t$ are odd integers $\geqq 3$. These are non-minimal critical points of the Yang-Mills functional, and we investigate the index and the nullity of the Hessian at these points. The nullity gives information about the moduli space of non-self-dual YM solutions, while the index gives topological information about the space of all connections, modulo gauge transformations.

Our investigation is numerical. We treat the Hessian one representation of $S O(3)$ at a time, and numerically diagonalize the Hessian in that representation, using a finite mode approximation to the space of deformations. We have results for $r$ and $t$ up to 13 , and $l$ up to 5 . We also apply the method to (anti)self-dual connections $(r, 1)$ and $(1, t)$, where the results are already known [BoSe], as a test of our method.

The numerical method is detailed in Sect. 5.1. The results are presented in Sect. 5.2. In Sect. 5.3 we discuss their significance.

5.1 The Numerical Method. Bor and Montgomery [BoMo] showed that, for a general smooth equivariant connection, the reduced connection $\left(a_{1}, a_{2}, a_{3}\right)$, which is naturally defined only on $[0, \pi / 3]$, can be extended to be a function on the entire circle. These functions have the following properties:

$$
a_{3}(\theta)=a_{3}(-\theta), \quad a_{2}(\theta)= \pm a_{3}(\theta+2 \pi / 3), \quad a_{1}(\theta)= \pm a_{3}(\theta-2 \pi / 3) .
$$

The signs in the second and third equations depend on whether $r$ and $t$ are congruent to $1(\bmod 4)$ or $3(\bmod 4)$.

The relations (5.1) essentially follow from the fact that, on $S^{4}$, the points $Q_{\theta}$, $Q_{-\theta}$ and $Q_{\theta \pm 2 \pi / 3}$ lie on the same orbit of the symmetry group $G=S O(3)$. The connection form at $Q_{-\theta}\left(\right.$ or $\left.Q_{\theta \pm 2 \pi / 3}\right)$ can either be written in terms of $a_{i}(-\theta)$, or as rotated versions of the connection form at $Q_{\theta}$.

So instead of working with three functions on the interval $[0, \pi / 3]$, we can work with the single function $a_{3}$ on the entire circle. Since this function is smooth, its Fourier coefficients decrease rapidly, and the function can be well-approximated with a finite Fourier series. This fact was used in [SS3] to get numerical approximations to the YM solutions for various values of $r$ and $t$. We minimized the YM functional in the space of functions whose Fourier expansions vanished after a fixed number of terms. Taking 10 terms gave remarkably good results in most cases, and taking 20 or 30 terms always gave the minimizing action to within one part in $10^{10}$.

The same trick of combining several functions on $[0, \pi / 3]$ into one function on the circle works with deformations. Since the coordinates $(\theta, g)$ and $(-\theta$, $\left.g \exp \left(\pi K_{3} / 2\right)\right)$ describe the same point on the sphere, we can decompose the connection at this point either in terms of $\alpha_{i j}^{m}(\theta)$ and $\gamma_{j}^{m}(\theta)$ or in terms of $\alpha_{i j}^{m}(-\theta)$ and $\gamma_{j}^{m}(-\theta)$. This, and similar equivalences between $\theta$ and $\theta \pm 2 \pi / 3$, gives relations similar to $(5.1)$.

For example, for $l=1$ a deformation can be written in terms of the 9 functions $\alpha_{32}^{1}, \alpha_{31}^{1}, \alpha_{23}^{1}, \alpha_{13}^{1}, \alpha_{12}^{0}, \alpha_{21}^{0}, \gamma_{1}^{1}, \gamma_{2}^{1}$, and $\gamma_{3}^{0}$ on $[0, \pi / 3]$. Alternatively, it can be written in terms of two functions, $\alpha$ and $\gamma$ on the circle. If $r \equiv t \equiv 3(\bmod 4)$, then

$$
\begin{aligned}
& \left.\alpha_{32}^{1}(\theta)=\alpha(\theta), \quad \alpha_{21}^{0}(\theta)=\alpha(\theta+2 \pi / 3), \quad \alpha_{13}^{1}(\theta)=\alpha(\theta-2 \pi / 3)\right), \\
& \alpha_{31}^{1}(\theta)=-\alpha(-\theta), \quad \alpha_{23}^{1}(\theta)=-\alpha(-\theta-2 \pi / 3), \quad \alpha_{12}^{0}(\theta)=-\alpha(-\theta+2 \pi / 3), \\
& \gamma_{3}^{0}(\theta)=\gamma(\theta)=-\gamma(-\theta), \quad \gamma_{2}^{1}(\theta)=\gamma(\theta+2 \pi / 3), \quad \gamma_{1}^{1}(\theta)=\gamma(\theta-2 \pi / 3) .
\end{aligned}
$$


If $r \equiv 1(\bmod 4)$ or $t \equiv 1(\bmod 4)$ then similar relations, with some signs changed, apply.

These functions of the circle can be Fourier decomposed. Since $\gamma$ is an odd function, it can be decomposed into sine functions, while $\alpha$ decomposes into both cosines and sines. For each frequency, we thus have 3 modes, two sines and a cosine, to consider. For larger values of $l$ we need more than 2 functions on the circle to describe $\delta A$, and in general we have $2 l+1$ modes associated to each frequency.

These Fourier modes form a natural basis for the space of deformations for each representation of $S O(3)$. We cut off this basis at a maximum frequency $N$ to get a finite-dimensional space. We compute the matrix elements of the Hessian (4.5) and of $\int\left[d_{A}^{*}(\delta A)\right]^{2}$ with respect to this basis, and then numerically diagonalize the matrix of $\delta^{2} S+\int\left(d_{A}^{*}(\delta A)\right)^{2}$.

There are three potential sources of error in this procedure, none of which actually cause problems. Round-off error in the computer gives errors of order of magnitude $10^{-14}$. The second source of error is our finite-mode approximation in calculating the reduced YM connection $\left(a_{1}, a_{2}, a_{3}\right)$. By taking enough Fourier components we limit this error to less than 1 part in $10^{6}$. This means that a zero eigenvalue may appear slightly negative (or positive), but will still be a factor of $10^{6}$ smaller than the other eigenvalues. Finally, there is our finite-mode approximation for the deformations. By restricting ourselves to a subspace of the space of deformations, we raise all the eigenvalues. In principle, this could mean that negative or zero modes could actually appear positive. In practice, however, this does not seem to occur. The positive and negative eigenvalues change only slightly when the highest allowed frequency, which we denote $N$, is increased, say from 20 to 30 . The eigenvalues close to zero shrink quickly as $N$ is increased, as is to be expected to true zero modes.

Table 1 shows the ten smallest eigenvalues, for $l=2$ and $N=20$, for two different critical points. One critical point has self-dual curvature, with $(r, t)=(1,5)$, while the second is non-self-dual, with $(r, t)=(5,3)$. The results are completely unambiguous. $(1,5)$ has one zero mode and no negative modes. $(5,3)$ has one zero mode and one negative mode.

5.2 Results. Let $h_{-}(l, r, t)$ be the dimension of the negative eigenspace of the Hessian for the $(r, t)$ YM connection, restricted to the spin-l representation of

Table 1. Lowest Eigenvalues for $l=2, N=20$

\begin{tabular}{lc}
\hline$(r, t)=(1,5)$ & $(r, t)=(5,3)$ \\
\hline 206.274560510209 & 171.127481544641 \\
162.883311525700 & 135.206418912372 \\
125.836315992737 & 122.690117492145 \\
120.723046719095 & 91.3946372008735 \\
115.713667988154 & 88.8817452481566 \\
84.6803226260188 & 80.0597577062249 \\
75.2720093738385 & 44.6778661554892 \\
47.6005044927976 & 38.3540729641350 \\
35.6509304053161 & $2.64380290969914 \times 10^{-10}$ \\
$2.06865281842209 \times 10^{-12}$ & -457.162248939746 \\
\hline
\end{tabular}


$S O(3)$. Let $h_{0}(l, r, t)$ be the dimension of the zero eigenspace. Of course, for (anti)self-dual connections $h_{-}(l, r, t)=0$ for all $l$.

The results for $h_{-}$, for $3 \leqq r, t \leqq 13$ are as follows:

1: $h_{-}(1, r, t)=1$.

2: $h_{-}(2, r, t)=1$.

3: $h_{-}(3, r, t)=2$ when $r$ and $t$ are both greater than $3 . h_{-}(3, r, t)=1$ when $r>t=3$ or $t>r=3$. $h_{-}(3,3,3)=0$.

4: $h_{-}(4, r, t)=h_{-}(3, r, t)$.

5: $h_{-}(5, r, t)=3$ when $r$ and $t$ are both greater than $5 . h_{-}(5, r, t)=2$ when $r>t=5$ or $t>r=5$. $h_{-}(5, r, t)=1$ when $r>5$ and $t=3$ or when $t>5$ and $r=3$ or when $r=t=5 . h_{-}(5,5,3)=h_{-}(5,3,5)=h_{-}(5,3,3)=0$.

These results suggest the following conjecture:

Conjecture 1. For any positive odd integers $r$ and $t$ and any positive integer $k$,

$$
h_{-}(2 k, r, t)=h_{-}(2 k-1, r, t)=P\left(\frac{2 k-P[2 k+1-r]-P[2 k+1-t]}{2}\right),
$$

where $P(x)=x$ when $x>0$ and 0 otherwise.

In other words, $h_{-}=k$ if both $r$ and $t$ are greater or equal to $2 k+1$, is reduced by one if $r=2 k-1$, is reduced by two if $r=2 k-3$, and so on, with similar reductions for the value of $t$. Note that, in addition to matching our numerical results for non-self-dual connections, formula (5.3) gives the correct dimension for the (anti)self-dual cases, namely zero for all $l$.

From conjecture 1 we derive the total index of the Hessian.

Index of Hessian of $(r, t)$ connection $=\sum_{l=0}^{\infty}(2 l+1) h_{-}(l, r, t)$

$$
\begin{aligned}
= & \sum_{k=1}^{\infty} 8 k P(2 k-P[2 k+1-r] \\
& -P[2 k+1-t]) / 2 \\
= & (r-1)(t-1)(r+t-2) / 2 .
\end{aligned}
$$

This index is always a multiple of 8 , and grows very quickly with increasing $r$ and $t$. The six smallest indices are $8,24,24,48,48$ and 64 , corresponding to the $(3,3),(5,3)$, $(3,5),(7,3),(3,7)$, and $(5,5)$ connections, respectively.

Taubes has shown [T1] that a NSD YM connection of second Chern number $C_{2}$ over $S^{4}$ must have Morse index at least $2\left|C_{2}\right|+2$. Conjecture 1 implies that equivariant NSD YM connections far exceed that lower bound. If $t=3$, formula (5.4) gives an index of $r^{2}-1$, four times the Taubes bound. Larger values of $t$ given an even greater discrepancy, since increasing $t$ increases the index but decreases the Chern number. In general, if $r \geqq t$, the index is at least $2(t-1)$ times larger than the Taubes bound.

We next turn to the nullity, where our results are extremely simple. For all the non-self-dual cases, $h_{0}(2, r, t)=h_{0}(3, r, t)=1$ and $h_{0}(1, r, t)=h_{0}(4, r, t)=$ $h_{0}(5, r, t)=0$. Since the $l=2$ and $l=3$ zero modes (and no others) are required by conformal symmetry, this suggests the following: 
Conjecture 2. For any positive odd integers $r \geqq 3$ and $t \geqq 3$, and for any $l, h_{0}(l, r, t)$ equals 1 if $l=2$ or 3 and is zero otherwise.

5.3 Discussion and Open Problems. Let $\mathcal{N}_{k}$ be the moduli space of all YM connections on an $S O(3)$ bundles over $S^{4}$ with second Chern number $-k$, modulo gauge transformations, and let $\mathscr{M}_{k} \subset \mathscr{N}_{k}$ be the moduli space of (anti)self-dual connections. Conjecture 2 implies that the component of $\mathscr{N}_{k}$ containing a non-self-dual $G$-equivariant YM connections consists only of conformal copies of that connection. Topologically, this component is the quotient of the conformal group $S O(4,1)$ by the subgroup $G=S O(3)$ that fixes the equivariant connection, and does not in any way depend on the values of $r$ and $t$.

There is a natural extension of conjecture 2 to non-equivariant connections, namely

Conjecture 3. Let $A_{1}$ and $A_{2}$ be non-self-dual YM connections over $S^{4}$ with second Chern number - $k$. Then either $A_{1}$ and $A_{2}$ lie in different components of $\mathscr{N}_{k}$, or $A_{1}$ is gauge-equivalent to a conformal copy of $A_{2}$.

At first glance, conjecture 3 is a disappointment. The non-self-dual components of the moduli space lack the rich structure of the (anti)self-dual component. However, conjecture 3 also says that non-self-dual YM connections are the closest thing possible to nondegenerate critical points of the YM functional. This makes it much easier to identify their topological role.

If we allow ourselves to vary the metric functions $f_{i}(\theta)$, we can even find true non-degenerate critical points. The proof of Theorem 3.1 uses only the asymptotic properties of the $f_{i}$, s, so the construction of NSD YM connections proceeds just as well with altered metric. The only difference is that, for a generic set of functions $f_{i}$, the conformal group is reduced from $S O(4,1)$ to $G=S O(3)$. By definition, each equivariant connection is left unchanged by $G$, so there are no conformal copies of a given equivariant NSD YM connection. We would then expect these NSD YM connections to be non-degenerate critical points of the YM functional.

We can get the same effect without changing the metric if we take $r$ or $t$ to be other than an odd integer. We then would be working on $S^{4}-S_{+}$rather than on $S^{4}$. Since the only conformal transformations of $S^{4}$ that leave $S_{+}$(or $S_{-}$) fixed are in $G$, the conformal group of $S^{4}-S_{ \pm}$is just $G$.

In either case, we would have a non-degenerate critical point. Such points are stable under small perturbations of the functional, and hence under small changes in the metric. These changes need not be equivariant. The $(r, t)$ NSD YM connections persist even when all symmetry has been broken. They have topological significance, and are not mere flukes of symmetry.

Some caution is in order, however. Hong-Yu Wang [W] has developed a technique for grafting a set of instanton-anti-instanton pairs onto some symmetric NSD YM connections on $S^{2} \times S^{2}$ and $S^{3} \times S^{1}$, thereby generating a non-conformal family of higher energy NSD YM connections. If this technique could be applied to the $(r, t)$ connections on $S^{4}$, it would provide a counter-example to conjecture 3 .

We now return to smooth bundles over $S^{4}$ with the round metric. Let $\mathscr{A}_{k}$ be the space of smooth $S O(3)$ connections with second Chern number $-k$, and let $\mathscr{G}$ be group of gauge transformations. The Yang-Mills functional is gauge-invariant, and so is a functional on $B_{k}=\mathscr{A}_{k} / \mathscr{G}$. We are interested in describing the topology of $B_{k}$, via Morse theory, by the critical points of the YM functional, i.e. by $\mathscr{N}_{k}$. 
Of course, Morse theory need not be exact, since the Yang-Mills functional in 4 dimensions does not satisfy the Palais-Smale condition. In the case of $B_{0}$, critical points of index 1 do not exist, as $1<2|k|+2=2$, yet Taubes explicitly found a non-contractible loop. This loop corresponds to what Taubes calls a critical end of $B_{k}$ [T2], not to a critical point. In general, the topology of $B_{k}$ is described partly by the minima of the YM functional $\left(\mathscr{M}_{k}\right)$, partly by the higher critical points (the rest of $\mathscr{N}_{k}$ ), and partly by critical ends. The obvious question is how much of the topology comes from each of these three contributions.

Atiyah and Jones [AJ] conjectured that $\mathscr{M}_{k}$ is homotopy equivalent to $B_{k}$ up through a range. The Atiyah-Jones conjecture was recently proven by Boyer et al. [BHMM1, BHMM2], who showed that $B_{k}$ and $\mathscr{M}_{k}$ are homotopy equivalent at least through dimension $[k / 2]-2$. Boyer et al. also found that, above the range of equivalence, $H_{*}\left(\mathscr{M}_{k}\right)$ has rational elements, while $H_{*}\left(B_{k}\right)$ is pure torsion. The higher critical points and the critical ends must not only provide the topology of $B_{k}$ that is not in $\mathscr{M}_{k}$, but must also cancel those elements of $H_{*}\left(\mathscr{M}_{k}\right)$ that are not in $H_{*}\left(B_{k}\right)$.

The space $B_{k}$ does not depend in any way on the metric, and the topology of $\mathscr{M}_{k}$ is also metric-invariant. However, the higher critical points and the critical ends very much depend on the metric. A change in metric can, by breaking conformal symmetry, convert a critical surface into a discrete set of critical points. A change in metric can also change a critical end into a critical point.

Parker [Pa] showed how, by changing the metric on $S^{4}$ an arbitrarily small amount, one can form a NSD YM connections with Chern number zero and with energy arbitrarily close to two instanton units. Parker's solution consists of a very small instanton and a very small anti-instanton, centered at antipodal points. If the metric were round, both the instanton and anti-instanton would bubble off under gradient flow. However, with the slightly altered metric, bubbling off is energetically unfavorable and the instanton and anti-instanton balance. Parker in effect raised the energy of Taubes' critical end and turned it into a critical point.

Presumably, the process can be repeated for other critical ends. It is unclear, however, whether there exist critical ends that cannot be so removed. We close with some open questions:

Questions. Given integers $i, q$ and $k$, do there exist metrics on $S^{4}$, arbitrarily close to the round metric in the $C^{q}$ norm, such that $B_{k}$ contains no critical ends with index less than i? If so, how large is the space of such metrics? Do there exist metrics that eliminate all critical ends?

Acknowledgements. I thank Gil Bor, Peter Kronheimer, Benjamin Mann, Jalal Shatah, Karen Uhlenbeck, and especially Jan Segert for helpful discussions. Many of the ideas about decomposing deformations of the connection into representations of $S O(3)$ are due to Segert. This work was partially supported by an NSF Mathematical Sciences Postdoctoral Fellowship.

\section{References}

[ADHM] Atiyah, M.F., Drinfeld, V.G., Hitchin, N.J., Manin, Y.I.: Construction of Instantons. Phys. Lett. 65A, 185-187 (1978)

[AJ] Atiyah, M.F., Jones, J.D.S.: Topological aspects of Yang-Mills theory. Commun. Math. Phys. 61, 97 (1978) 
[BHMM1] Boyer, C.P., Hurtubise, J.C., Mann, B.M., Milgram, R.J.: The Atiyah-Jones conjecture. Bull. Am. Math. Soc. 26, 317-321 (1992)

[BHMM2] Boyer, C.P., Hurtubise, J.C., Mann, B.M., Milgram, R.J.: The topology of instanton moduli spaces I: The Atiyah-Jones conjecture. Ann. Math. 137, 561-609 (1993)

[BoMo] Bor, G., Montgomery, R.: SO(3) Invariant Yang-Mills Fields Which Are Not SelfDual. In: Harnad, J., Marsden, J.E. (eds.) Hamiltonian Systems, Transformation Groups, and Spectral Transform Methods. Proceedings, Montreal, 1989, Montreal: Les publications CRM, 1990

[Bor] Bor, G.: Yang-Mills Fields which are not Self-Dual. Commun. Math. Phys. 145, 393-410 (1992)

[BoSe] Bor, G., Segert, J.: Rational solutions of the quadrupole self-duality equation. Preprint, 1993

[DK] Donaldson, S.K., Kronheimer, P.B.: The geometry of four-manifolds. Oxford: Oxford University Press, 1990

[FHP1] Forgacs, P., Horvath, Z., Palla, L.: An exact fractionally charged self-dual solution. Phys. Rev. Lett. 46, 392 (1981)

[FHP2] Forgacs, P., Horvath, Z., Palla, L.: One Can Have Noninteger Topological Charge. Z. Phys. C - Particles and Fields 12, 359-360 (1982)

[K] Kronheimer, P.B.: Embedded surfaces in 4-manifolds. Proceedings of the International Congress of mathematicians (Kyoto 1990), Tokyo Berlin, 1991

[KM] Kronheimer, P.B., Mrowka, T.S.: Gauge theory for embedded surfaces I. Topology 32, 773-826 (1993)

[Pa] Parker, T.: Non-minimal Yang-Mills Fields and Dynamics. Invent. Math. 107, 397-420 (1992)

[R1] Råde, J.: Singular Yang-Mills Fields. Local theory I. J. reine angew. Math. (in press)

[R2] Råde, J.: Singular Yang-Mills Fields. Local theory II. J. reine angew. Math. (in press)

[SS1] Sadun, L. Segert, J.: Non-Self-Dual Yang-Mills connections with nonzero Chern number. Bull. Am. Math. Soc. 24, 163-170 (1991)

[SS2] Sadun, L., Segert, J.: Non-Self-Dual Yang-Mills connections with Quadrupole Symmetry. Commun. Math. Phys. 145, 363-391 (1992)

[SS3] Sadun, L., Segert, J.: Stationary points of the Yang-Mills action. Commun. Pure Appl. Math. 45, 461-484 (1992)

[SiSi1] Sibner, L.M., Sibner, R.J.: Singular Soblev Connections with Holonomy. Bull. Am. Math. Soc. 19, 471-473 (1988)

[SiSi2] Sibner, L.M., Sibner, R.J.: Classification of Singular Sobolev Connections by their Holonomy. Commun. Math. Phys. 144, 337-350 (1992)

[SSU] Sibner, L.M., Sibner, R.J., Uhlenbeck, K.: Solutions to Yang-Mills Equations which are not Self-Dual. Proc. Natl. Acad. Sci. USA 86, 8610-8613 (1989)

[T1] Taubes, C.H.: Stability in Yang-Mills theories. Comm. Math. Phys. 91, 235-263 (1983)

[T2] Taubes, C.H.: A framework for Morse theory for the Yang-Mills functional. Invent. Math. 94, 327-402 (1988)

[Ur] Urakawa, H.: Equivariant Theory of Yang-Mills Connections over Riemannian Manifolds of Cohomogeneity One. Indiana Univ. Math. J. 37, 753-788 (1988)

[W] Hong-Yu Wang: The existence of non-minimal solutions to the Yang-Mills equation with group $S U(2)$ on $S^{2} \times S^{2}$ and $S^{1} \times S^{3}$. J. Diff. Geom. 34, 701-767 (1991) 
